Finite Fields Appl. 59 (2019), 246-283.

\title{
QUADRATIC RESIDUES AND RELATED PERMUTATIONS AND IDENTITIES
}

\author{
ZHI-WEI SUN \\ Department of Mathematics, Nanjing University \\ Nanjing 210093, People's Republic of China \\ zwsun@nju.edu.cn \\ http://math.nju.edu.cn/ zwsun
}

\begin{abstract}
Let $p$ be an odd prime. In this paper we investigate quadratic residues modulo $p$ and related permutations, congruences and identities. If $a_{1}<\ldots<a_{(p-1) / 2}$ are all the quadratic residues modulo $p$ among $1, \ldots, p-1$, then the list $\left\{1^{2}\right\}_{p}, \ldots,\left\{((p-1) / 2)^{2}\right\}_{p}$ (with $\{k\}_{p}$ the least nonnegative residue of $k$ modulo $p$ ) is a permutation of $a_{1}, \ldots, a_{(p-1) / 2}$, and we show that the sign of this permutation is 1 or $(-1)^{(h(-p)+1) / 2}$ according as $p \equiv 3(\bmod 8)$ or $p \equiv 7(\bmod 8)$, where $h(-p)$ is the class number of the imaginary quadratic field $\mathbb{Q}(\sqrt{-p})$. To achieve this, we evaluate the product $\prod_{1 \leqslant j<k \leqslant(p-1) / 2}\left(\cot \pi j^{2} / p-\right.$ $\left.\cot \pi k^{2} / p\right)$ via Dirichlet's class number formula and Galois theory. We also obtain some new congruences and identities in product forms; for example, we
\end{abstract} determine the exact value of

$$
\prod_{1 \leqslant j<k \leqslant p-1} \cos \pi \frac{a j^{2}+b j k+c k^{2}}{p}
$$

for any $a, b, c \in \mathbb{Z}$ with $a c(a+b+c) \not \equiv 0(\bmod p)$.

\section{INTRODUCTION}

Let $n$ be any positive integer. A permutation $\sigma$ on the set $\{1, \ldots, n\}$ is said to be odd or even according as

$$
\operatorname{Inv}(\sigma):=\mid\{(i, j): 1 \leqslant i<j \leqslant n \text { and } \sigma(i)>\sigma(j)\} \mid
$$

is odd or even. The sign of the permutation $\sigma$ is given by $\operatorname{sign}(\sigma)=(-1)^{\operatorname{Inv}(\sigma)}$. For integers $a$ and $b \neq 0$ with $\operatorname{gcd}(b, n)=1$, we use $\{a / b\}_{n}$ to denote the unique integer $r \in\{0, \ldots, n-1\}$ with $a / b \equiv r(\bmod n)$ (i.e., $a \equiv b r(\bmod n))$.

2010 Mathematics Subject Classification. Primary 11A15, 05A05, 11T24, 33B10; Secondary 11A07, 11R32.

Keywords. Congruences modulo primes, permutations, quadratic residues, Galois theory, Gauss sums.

Supported by the National Natural Science Foundation of China (grant no. 11571162) and the NSFC-RFBR Cooperation and Exchange Program (grant no. 11811530072). 
Let $p$ be an odd prime and let $a \in \mathbb{Z}$ with $p \nmid a$. Then $\pi_{a}(k)=\{a k\}_{p}$ with $1 \leqslant k \leqslant p-1$ is a permutation on $\{1, \ldots, p-1\}$. Zolotarev's lemma (cf. [DH] and $[\mathrm{Z}])$ asserts that $\operatorname{sign}\left(\pi_{a}\right)$ coincides with the Legendre symbol $\left(\frac{a}{p}\right)$.

Frobenius (cf. [BC]) extended Zolotarev's lemma as follows: If $a \in \mathbb{Z}$ is relatively prime to a positive odd integer $n$, then the sign of the permutation $\pi_{a}(k)=\{a k\}_{n}(0 \leqslant k \leqslant n-1)$ on $\{0, \ldots, n-1\}$ equals the Jacobi symbol $\left(\frac{a}{n}\right)$.

Let $n>1$ be an odd integer and let $a$ be any integer relatively prime to $n$. For each $k=1, \ldots,(n-1) / 2$ let $\pi_{a}^{*}(k)$ be the unique $r \in\{1, \ldots,(n-1) / 2\}$ with $a k$ congruent to $r$ or $-r$ modulo $n$. For the permutation $\pi_{a}^{*}$ on $\{1, \ldots,(n-1) / 2\}$, Pan [P06] showed that its sign is given by

$$
\operatorname{sign}\left(\pi_{a}^{*}\right)=\left(\frac{a}{n}\right)^{(n+1) / 2}
$$

Let $m>1$ be an odd integer, and let $a_{1}<\ldots<a_{\varphi(m)}$ be all the numbers among $1, \ldots, m-1$ relatively prime to $m$. For each $k \in\{1, \ldots, m-1\}$ with $\operatorname{gcd}(k, m)=1$, let $\sigma_{m}(k)=\bar{k}$ be the inverse of $k$ modulo $m$, that is, $\bar{k} \in$ $\{1, \ldots, m-1\}$ and $k \bar{k} \equiv 1(\bmod m)$. For $k=1, \ldots,(m-1) / 2$ with $\operatorname{gcd}(k, m)=$ 1 , let $\tau_{m}(k)$ be the unique integer $k^{*} \in\{1, \ldots,(m-1) / 2\}$ such that $k k^{*}$ is congruent to 1 or -1 modulo $m$. Clearly, $\sigma_{m}$ is a permutation of $a_{1}, \ldots, a_{\varphi(m)}$, and $\tau_{m}$ is the permutation of $a_{1}, \ldots, a_{\varphi(m) / 2}$. Our first theorem determines $\operatorname{sign}\left(\sigma_{m}\right)$ and $\operatorname{sign}\left(\tau_{m}\right)$.

Theorem 1.1. Suppose that $m=\prod_{s=1}^{r} p_{s}^{a_{s}}$, where $p_{1}, \ldots, p_{r}$ are distinct odd primes and $a_{1}, \ldots, a_{r}$ are positive integers. Then we have

$$
\operatorname{sign}\left(\sigma_{m}\right)=-1 \Longleftrightarrow r=1 \text { and } p_{1} \equiv 1 \quad(\bmod 4)
$$

Also, $\operatorname{sign}\left(\tau_{m}\right)=-1$ if and only if $r=1 \&\left(p_{1} \equiv 1\right.$ or $\left.4 a_{1}+3(\bmod 8)\right)$, or $\left(r=2 \& p_{1}+p_{2} \equiv 0(\bmod 4)\right)$. In particular, when $m$ is an odd prime we have

$$
\operatorname{sign}\left(\sigma_{m}\right)=-\left(\frac{-1}{m}\right) \text { and } \operatorname{sign}\left(\tau_{m}\right)=-\left(\frac{2}{m}\right) .
$$

Let $p$ be an odd prime. By Wilson's theorem,

$$
(-1)^{(p-1) / 2}\left(\frac{p-1}{2} !\right)^{2} \equiv \prod_{k=1}^{(p-1) / 2} k(p-k)=(p-1) ! \equiv-1 \quad(\bmod p) .
$$

Write $p=2 n+1$ and let $a_{1}, \ldots, a_{n}$ be the list of all the $n$ quadratic residues among $1, \ldots, p-1$ in the ascending order. It is well known that the list

$$
\left\{1^{2}\right\}_{p}, \ldots,\left\{n^{2}\right\}_{p}
$$


is a permutation of $a_{1}, \ldots, a_{n}$. Clearly, the sign of this permutation is just the sign of the product

$$
S_{p}:=\prod_{1 \leqslant i<j \leqslant(p-1) / 2}\left(\left\{j^{2}\right\}_{p}-\left\{i^{2}\right\}_{p}\right)
$$

(An empty product like $S_{3}$ is regarded to have the value 1.) It is easy to determine this product modulo $p$. In fact,

$$
\prod_{1 \leqslant i<j \leqslant n}\left(j^{2}-i^{2}\right) \equiv \begin{cases}-n !(\bmod p) & \text { if } p \equiv 1(\bmod 4) \\ 1(\bmod p) & \text { if } p \equiv 3(\bmod 4)\end{cases}
$$

because

$$
\begin{aligned}
\prod_{1 \leqslant i<j \leqslant n}(j-i) \times \prod_{1 \leqslant i<j \leqslant n}(j+i) & =\prod_{k=1}^{n} k^{|\{i \geqslant 1: k+i \leqslant n\}|} \times \prod_{k=1}^{p-1} k^{|\{1 \leqslant i<k / 2: k-i \leqslant n\}|} \\
& =\prod_{k=1}^{n} k^{n-k} \times \prod_{k=1}^{n} k^{\lfloor(k-1) / 2\rfloor}(p-k)^{\lfloor k / 2\rfloor} \\
& \equiv(-1)^{\sum_{k=0}^{n}\lfloor k / 2\rfloor}(n !)^{n-1}(\bmod p)
\end{aligned}
$$

and $(n !)^{2} \equiv(-1)^{n+1}(\bmod p)$ by $(1.3)$. Note that if $p \equiv 3(\bmod 4)$ then

$$
\prod_{1 \leqslant i<j \leqslant(p-1) / 2}\left(i^{2}+j^{2}\right) \equiv(-1)^{\lfloor(p+1) / 8\rfloor} \quad(\bmod p)
$$

(cf. Problem N.2 of [Sz, pp. 364-365]).

Inspired by (1.5) and (1.6), we obtain the following general result.

Theorem 1.2. Let $p$ be an odd prime.

(i) If $p \equiv 1(\bmod 4)$, then

$$
\prod_{\substack{1 \leqslant i<j \leqslant(p-1) / 2 \\ p \nmid i^{2}+j^{2}}}\left(i^{2}+j^{2}\right) \equiv(-1)^{\lfloor(p-5) / 8\rfloor}(\bmod p) .
$$

(ii) Let $a, b, c \in \mathbb{Z}$ with $a c(a+b+c) \not \equiv 0(\bmod p)$, and set $\Delta=b^{2}-4 a c$. Then

$$
\prod_{\substack{1 \leqslant i<j \leqslant p-1 \\ p \nmid a i^{2}+b i j+c j^{2}}}\left(a i^{2}+b i j+c j^{2}\right) \equiv \begin{cases}\left(\frac{a(a+b+c)}{p}\right)(\bmod p) & \text { if } p \mid \Delta, \\ -\left(\frac{a c(a+b+c) \Delta}{p}\right)(\bmod p) & \text { if } p \nmid \Delta .\end{cases}
$$


If $a+c=0$, then

$$
\begin{gathered}
\prod_{\substack{i, j=1 \\
p \nmid a i^{2}+b i j+c j^{2}}}^{(p-1) / 2}\left(a i^{2}+b i j+c j^{2}\right) \\
\equiv \begin{cases} \pm \frac{p-1}{2} !(\bmod p) & \text { if }\left(\frac{\Delta}{p}\right)=-1 \text { or }\left(p \mid \Delta \&\left(\frac{2 b}{p}\right)=1\right), \\
\pm 1(\bmod p) & \text { if }\left(\frac{\Delta}{p}\right)=1 \text { or }\left(p \mid \Delta \&\left(\frac{2 b}{p}\right)=-1\right) .\end{cases}
\end{gathered}
$$

(iii) Let $a, b, c \in \mathbb{Z}$ with $p \nmid a c$ and $p \mid a+b+c$. Then

$$
\prod_{\substack{1 \leqslant i<j \leqslant p-1 \\ p \nmid a i^{2}+b i j+c j^{2}}}\left(a i^{2}+b i j+c j^{2}\right) \equiv \begin{cases}(-1)^{N_{p}(a / c)}\left(\frac{2 c(a-c)}{p}\right)(\bmod p) & \text { if } p \nmid a-c, \\ (-1)^{(p+1) / 2}\left(\frac{a}{p}\right)(\bmod p) & \text { if } p \mid a-c,\end{cases}
$$

where $N_{p}(x):=\left|\left\{1 \leqslant k \leqslant(p-1) / 2:\{k x\}_{p}>k\right\}\right|$ for any $p$-adic integer $x$.

(iv) Let $a, b, c \in \mathbb{Z}$ with $p \mid a c$. Then

$$
\begin{gathered}
\prod_{\substack{1 \leqslant i<j \leqslant p-1 \\
p \nmid a i^{2}+b i j+c j^{2}}}\left(a i^{2}+b i j+c j^{2}\right) \\
\equiv \begin{cases}-\left(\frac{b}{p}\right)(\bmod p) & \text { if } p \mid a, p \nmid b \text { and } p \mid c, \\
-\left(\frac{c}{p}\right)(\bmod p) & \text { if } p \mid a, p \nmid b c \text { and } p \mid b+c, \\
(-1)^{N_{p}(-c / b)}\left(\frac{2}{p}\right)(\bmod p) & \text { if } p \mid a \text { and } p \nmid b c(b+c), \\
(-1)^{(p+1) / 2}\left(\frac{c}{p}\right)(\bmod p) & \text { if } p|a, p| b \text { and } p \nmid c, \\
(-1)^{(p+1) / 2}\left(\frac{a}{p}\right)(\bmod p) & \text { if } p \nmid a, p \mid b \text { and } p \mid c, \\
(-1)^{(p+1) / 2}\left(\frac{b}{p}\right)(\bmod p) & \text { if } p \nmid a b, p \mid a+b \text { and } p \mid c, \\
(-1)^{N_{p}(-a / b)}\left(\frac{2}{p}\right)(\bmod p) & \text { if } p \nmid a b(a+b) \text { and } p \mid c .\end{cases}
\end{gathered}
$$

To determine the sign of $S_{p}$ for an arbitrary prime $p \equiv 3(\bmod 4)$, we need to establish the following theorem via Dirichlet's class number formula and Galois theory.

Theorem 1.3. Let $p>3$ be a prime and let $\zeta=e^{2 \pi i / p}$. Let a be any integer not divisible by $p$.

(i) If $p \equiv 1(\bmod 4)$, then

$$
\prod_{k=1}^{(p-1) / 2}\left(1-\zeta^{a k^{2}}\right)=\sqrt{p} \varepsilon_{p}^{-\left(\frac{a}{p}\right) h(p)}
$$


where $\varepsilon_{p}$ and $h(p)$ are the fundamental unit and the class number of the real quadratic field $\mathbb{Q}(\sqrt{p})$ respectively. If $p \equiv 3(\bmod 4)$, then

$$
\prod_{k=1}^{(p-1) / 2}\left(1-\zeta^{a k^{2}}\right)=(-1)^{(h(-p)+1) / 2}\left(\frac{a}{p}\right) \sqrt{p} i .
$$

(ii) If $p \equiv 1(\bmod 4)$, then

$$
\prod_{1 \leqslant j<k \leqslant(p-1) / 2}\left(\zeta^{a j^{2}}-\zeta^{a k^{2}}\right)^{2}=(-1)^{(p-1) / 4} p^{(p-3) / 4} \varepsilon_{p}^{\left(\frac{a}{p}\right) h(p)} .
$$

When $p \equiv 3(\bmod 4)$, we have

$$
\begin{aligned}
& \prod_{1 \leqslant j<k \leqslant(p-1) / 2}\left(\zeta^{a j^{2}}-\zeta^{a k^{2}}\right) \\
= & \begin{cases}(-p)^{(p-3) / 8} & \text { if } p \equiv 3(\bmod 8), \\
(-1)^{(p+1) / 8+(h(-p)-1) / 2}\left(\frac{a}{p}\right) p^{(p-3) / 8} i & \text { if } p \equiv 7(\bmod 8),\end{cases}
\end{aligned}
$$

where $h(-p)$ is the class number of the imaginary quadratic field $\mathbb{Q}(\sqrt{-p})$.

Remark 1.1. For any prime $p \equiv 3(\bmod 4)$, it is known that $2 \nmid h(-p)$; moreover, L. J. Mordell [M61] proved that $\frac{p-1}{2} ! \equiv(-1)^{(h(-p)+1) / 2}(\bmod p)$ if $p>3$. In the case $a=1$, Theorem 1.3(i) appeared in [Ch]. Our proof of (1.15) utilizes the congruence (1.5).

Since

$$
\sin \pi \theta=\frac{i}{2} e^{-i \pi \theta}\left(1-e^{2 \pi i \theta}\right) \quad \text { and } \quad 2 \cos \pi \theta \times \sin \pi \theta=\sin 2 \pi \theta,
$$

we can easily deduce the following corollary from Theorem 1.3(i).

Corollary 1.1. Let $p>3$ be a prime and let $a \in \mathbb{Z}$ with $p \nmid a$. Then

$$
\begin{aligned}
& 2^{(p-1) / 2} \prod_{k=1}^{(p-1) / 2} \sin \pi \frac{a k^{2}}{p} \\
= & (-1)^{(a+1)\lfloor(p+1) / 4\rfloor} \sqrt{p} \times \begin{cases}\varepsilon_{p}^{-\left(\frac{a}{p}\right) h(p)} & \text { if } p \equiv 1(\bmod 4), \\
(-1)^{(h(-p)+1) / 2}\left(\frac{a}{p}\right) & \text { if } p \equiv 3(\bmod 4),\end{cases}
\end{aligned}
$$

and

$$
2^{(p-1) / 2} \prod_{k=1}^{(p-1) / 2} \cos \pi \frac{a k^{2}}{p}= \begin{cases}(-1)^{a(p-1) / 4} \varepsilon_{p}^{\left(1-\left(\frac{2}{p}\right)\right)\left(\frac{a}{p}\right) h(p)} & \text { if } p \equiv 1(\bmod 4) \\ (-1)^{(a+1)(p+1) / 4} & \text { if } p \equiv 3(\bmod 4) .\end{cases}
$$


For any odd prime $p$, we define

$$
s(p):=\mid\left\{(j, k): 1 \leqslant j<k \leqslant \frac{p-1}{2} \text { and }\left\{j^{2}\right\}_{p}>\left\{k^{2}\right\}_{p}\right\} \mid
$$

and

$$
t(p):=\mid\left\{(j, k): 1 \leqslant j<k \leqslant \frac{p-1}{2} \text { and }\left\{k^{2}-j^{2}\right\}_{p}>\frac{p}{2}\right\} \mid .
$$

For example, $s(11)=t(11)=4$ since $\left(\left\{1^{2}\right\}_{11}, \ldots,\left\{5^{2}\right\}_{11}\right)=(1,4,9,5,3)$,

$$
\left\{(j, k): 1 \leqslant j<k \leqslant 5 \&\left\{j^{2}\right\}_{11}>\left\{k^{2}\right\}_{11}\right\}=\{(2,5),(3,4),(3,5),(4,5)\},
$$

and

$$
\left\{(j, k): 1 \leqslant j<k \leqslant 5 \&\left\{k^{2}-j^{2}\right\}_{11}>\frac{11}{2}\right\}=\{(1,3),(2,5),(3,4),(4,5)\} .
$$

From Theorem 1.3 we deduce the following result.

Theorem 1.4. Let $p$ be an odd prime.

(i) We have

$$
\operatorname{sign}\left(S_{p}\right)=(-1)^{s(p)}=(-1)^{t(p)}= \begin{cases}1 & \text { if } p \equiv 3(\bmod 8), \\ (-1)^{(h(-p)+1) / 2} & \text { if } p \equiv 7(\bmod 8) .\end{cases}
$$

(ii) Let $a \in \mathbb{Z}$ with $p \nmid a$. Then

$$
\begin{aligned}
& \prod_{1 \leqslant j<k \leqslant(p-1) / 2} \csc \pi \frac{a\left(k^{2}-j^{2}\right)}{p}=\prod_{1 \leqslant j<k \leqslant(p-1) / 2}\left(\cot \pi \frac{a j^{2}}{p}-\cot \pi \frac{a k^{2}}{p}\right) \\
= & \begin{cases}\left(2^{p-1} / p\right)^{(p-3) / 8} & \text { if } p \equiv 3(\bmod 8), \\
(-1)^{(h(-p)+1) / 2}\left(\frac{a}{p}\right)\left(2^{p-1} / p\right)^{(p-3) / 8} & \text { if } p \equiv 7(\bmod 8) .\end{cases}
\end{aligned}
$$

In the case $p \equiv 1(\bmod 4)$, we have

$$
\begin{aligned}
& (-1)^{(a-1)(p-1) / 4} \prod_{1 \leqslant j<k \leqslant(p-1) / 2} \csc \pi \frac{a\left(k^{2}-j^{2}\right)}{p} \\
= & \varepsilon_{p}^{-\left(\frac{a}{p}\right) h(p)(p-1) / 2} \prod_{1 \leqslant j<k \leqslant(p-1) / 2}\left(\cot \pi \frac{a j^{2}}{p}-\cot \pi \frac{a k^{2}}{p}\right) \\
= & \pm\left(2^{p-1} p^{-1}\right)^{(p-3) / 8} \varepsilon_{p}^{-\left(\frac{a}{p}\right) h(p) / 2} .
\end{aligned}
$$

Remark 1.2. The values of $s(p)$ for the first 2500 odd primes $p$ are available from [S18, A319311]. That $2 \mid s(p)$ for any prime $p \equiv 3(\bmod 8) \operatorname{might}$ have a combinatorial proof.

With the help of Theorem 1.4, we also get the following result. 
Theorem 1.5. Let $p$ be an odd prime and let $\zeta=e^{2 \pi i / p}$. Let $a \in \mathbb{Z}$ with $p \nmid a$. Then

$$
\begin{aligned}
& (-1)^{a \frac{p+1}{2}\left\lfloor\frac{p-1}{4}\right\rfloor} 2^{(p-1)(p-3) / 8} \prod_{1 \leqslant j<k \leqslant(p-1) / 2} \cos \pi \frac{a\left(k^{2}-j^{2}\right)}{p} \\
= & \prod_{1 \leqslant j<k \leqslant(p-1) / 2}\left(\zeta^{a j^{2}}+\zeta^{a k^{2}}\right)= \begin{cases}1 & \text { if } p \equiv 3(\bmod 4), \\
\pm \varepsilon_{p}^{\left(\frac{a}{p}\right) h(p)\left(\left(\frac{2}{p}\right)-1\right) / 2} & \text { if } p \equiv 1(\bmod 4) .\end{cases}
\end{aligned}
$$

For a real number $x$ let $\{x\}$ denote its fractional part $x-\lfloor x\rfloor$. If $p$ is an odd prime and $1 \leqslant j<k \leqslant(p-1) / 2$, then

$$
\begin{aligned}
\cos 2 \pi \frac{k^{2}-j^{2}}{p}<0 & \Longleftrightarrow \cos 2 \pi\left|\left\{\frac{k^{2}}{p}\right\}-\left\{\frac{j^{2}}{p}\right\}\right|<0 \\
& \Longleftrightarrow \frac{1}{4}<\left|\left\{\frac{k^{2}}{p}\right\}-\left\{\frac{j^{2}}{p}\right\}\right|<\frac{3}{4} .
\end{aligned}
$$

Thus Theorem 1.5 with $a=2$ yields the following corollary.

Corollary 1.2. For any prime $p \equiv 3(\bmod 4)$, we have

$$
\mid\left\{(j, k): 1 \leqslant j<k \leqslant \frac{p-1}{2} \text { and } \frac{1}{4}<\left|\left\{\frac{k^{2}}{p}\right\}-\left\{\frac{j^{2}}{p}\right\}\right|<\frac{3}{4}\right\} \mid \equiv 0 \quad(\bmod 2) .
$$

Motivated by the congruences (1.5)-(1.6) and Theorems 1.2-1.5, we establish the following theorem.

Theorem 1.6. Let $p$ be an odd prime.

(i) Let $a \in \mathbb{Z}$ with $p \nmid a$. Then

$$
\begin{gathered}
\prod_{\substack{1 \leqslant j<k \leqslant(p-1) / 2 \\
p \nmid j^{2}+k^{2}}} \sin \pi \frac{a\left(j^{2}+k^{2}\right)}{p} \\
=\left(\frac{p}{2^{p-1}}\right)^{\left(p-\left(\frac{-1}{p}\right)-4\right) / 8} \times \begin{cases}\varepsilon_{p}^{\left(\frac{a}{p}\right) h(p)\left(1+\left(\frac{2}{p}\right)\right) / 2} & \text { if } p \equiv 1(\bmod 4), \\
(-1)^{(p-3) / 8} & \text { if } p \equiv 3(\bmod 8), \\
(-1)^{(p+1) / 8+(h(-p)+1) / 2}\left(\frac{a}{p}\right) & \text { if } p \equiv 7(\bmod 8) .\end{cases}
\end{gathered}
$$

Also,

$$
\prod_{1 \leqslant j<k \leqslant(p-1) / 2} \cos \pi \frac{a\left(j^{2}+k^{2}\right)}{p}=(-1)^{a \frac{p+1}{2}\left\lfloor\frac{p-1}{4}\right\rfloor} 2^{-\frac{p-1}{2}\left\lfloor\frac{p-3}{4}\right\rfloor}
$$


and

$$
\begin{gathered}
\prod_{\substack{1 \leqslant j<k \leqslant(p-1) / 2 \\
p \nmid j^{2}+k^{2}}}\left(\cot \pi \frac{a j^{2}}{p}+\cot \pi \frac{a k^{2}}{p}\right) \\
=\left(2^{p-1} p^{-1}\right)^{\left(p-\left(\frac{-1}{p}\right)-4\right) / 8} \times \begin{cases}\varepsilon_{p}^{\left(\frac{a}{p}\right) h(p)\left(p+\left(\frac{2}{p}\right)-4\right) / 2} & \text { if } p \equiv 1(\bmod 4), \\
(-1)^{(p-3) / 8} & \text { if } p \equiv 3(\bmod 8), \\
(-1)^{(p+1) / 8+(h(-p)+1) / 2}\left(\frac{a}{p}\right) & \text { if } p \equiv 7(\bmod 8) .\end{cases}
\end{gathered}
$$

(ii) Let $a, b, c \in \mathbb{Z}$ with $a c(a+b+c) \not \equiv 0(\bmod p)$. Set $\Delta=b^{2}-4 a c$ and

$$
m=\sum_{\substack{1 \leqslant j<k \leqslant p-1 \\ p \mid a j^{2}+b j k+c k^{2}}}\left(a j^{2}+b j k+c k^{2}\right) .
$$

Then

$$
\begin{aligned}
& (-1)^{m}\left(2^{p-1} p^{-1}\right)^{\left(p-3-\left(\frac{\Delta}{p}\right)\right) / 2} \prod_{\substack{1 \leqslant j<k \leqslant p-1 \\
p \nmid a j^{2}+b j k+c k^{2}}} \sin \pi \frac{a j^{2}+b j k+c k^{2}}{p}
\end{aligned}
$$

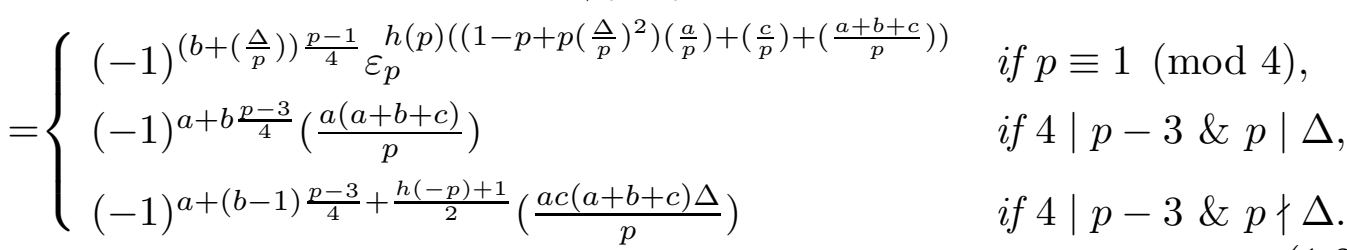

We also have

$$
\begin{aligned}
& 2^{(p-1)\left(p-3-\left(\frac{\Delta}{p}\right)\right) / 2} \prod_{1 \leqslant j<k \leqslant p-1} \cos \pi \frac{a j^{2}+b j k+c k^{2}}{p} \\
= & \begin{cases}(-1)^{b(p-1) / 4} \varepsilon_{p}^{h(p)\left(\left(\frac{2}{p}\right)-1\right)\left(\left(1-p+p\left(\frac{\Delta}{p}\right)^{2}\right)\left(\frac{a}{p}\right)+\left(\frac{c}{p}\right)+\left(\frac{a+b+c}{p}\right)\right)} & \text { if } p \equiv 1(\bmod 4), \\
(-1)^{a+b(p-3) / 4+\left(\frac{\Delta}{p}\right)(p+1) / 4} & \text { if } p \equiv 3(\bmod 4) .\end{cases}
\end{aligned}
$$

Remark 1.3. Under the notation in Theorem 1.6, as $4 a\left(a j^{2}+b j k+c k^{2}\right)=$ $(2 a j+b k)^{2}-\Delta k^{2}$ we have $m=0$ in the case $\left(\frac{\Delta}{p}\right)=-1$. It seems sophisticated to determine the parity of $m$ in the case $\left(\frac{\Delta}{p}\right) \geqslant 0$.

Let $p$ be any odd prime and let $a \in \mathbb{Z}$ with $p \nmid a$. For $1 \leqslant j<k \leqslant(p-1) / 2$, clearly

$$
\begin{aligned}
\left\{a j^{2}\right\}_{p}+\left\{a k^{2}\right\}_{p}>p & \Longleftrightarrow\left\{a j^{2}\right\}_{p}>\left\{-a k^{2}\right\}_{p} \\
& \Longleftrightarrow \cot \pi \frac{a j^{2}}{p}<\cot \pi \frac{-a k^{2}}{p} \\
& \Longleftrightarrow \cot \pi \frac{a j^{2}}{p}+\cot \pi \frac{a k^{2}}{p}<0 .
\end{aligned}
$$


QUADRATIC RESIDUES AND RELATED PERMUTATIONS AND IDENTITIES 9

Thus (1.27) yields the following consequence.

Corollary 1.3. Let $p$ be an odd prime and let $a \in \mathbb{Z}$ with $p \nmid a$. For

$$
N:=\left|\left\{(j, k): 1 \leqslant j<k \leqslant \frac{p-1}{2}:\left\{a j^{2}\right\}_{p}+\left\{a k^{2}\right\}_{p}>p\right\}\right|,
$$

we have

$$
(-1)^{N}= \begin{cases}1 & \text { if } p \equiv 1(\bmod 4), \\ (-1)^{(p-3) / 8} & \text { if } p \equiv 3(\bmod 8), \\ (-1)^{(p+1) / 8+(h(-p)+1) / 2}\left(\frac{a}{p}\right) & \text { if } p \equiv 7(\bmod 8) .\end{cases}
$$

We are going to show Theorems 1.1-1.2, Theorem 1.3, Theorems 1.4-1.5 and Theorem 1.6 in Sections 2-5 respectively. In Section 6 we pose some conjectures for further research.

\section{Proofs of Theorems 1.1-1.2}

Lemma 2.1. Suppose that $m=\prod_{s=1}^{r} p_{s}^{a_{s}}$, where $p_{1}, \ldots, p_{r}$ are distinct odd primes and $a_{1}, \ldots, a_{r}$ are positive integers. Then

$$
\mid\left\{1 \leqslant k<\frac{m}{2}: \operatorname{gcd}(k, m)=1 \text { and } \bar{k}<\frac{m}{2}\right\} \mid \equiv \delta_{r, 1} \quad(\bmod 2),
$$

where $\bar{k}$ is inverse of $k$ modulo $m$ (i.e., $1 \leqslant k \leqslant m-1$ and $k \bar{k} \equiv 1(\bmod m)$ ), and $\delta_{r, 1}$ is 1 or 0 according as $r=1$ or not. Also, the number

$$
M:=\mid\left\{(i, j): 1 \leqslant i<j<\frac{m}{2} \text { and } i j \equiv \pm 1(\bmod m)\right\} \mid
$$

is odd if and only if $r=1 \&\left(p_{1} \equiv 1\right.$ or $\left.4 a_{1}+3(\bmod 8)\right)$, or $\left(r=2 \& p_{1}+p_{2} \equiv 0\right.$ $(\bmod 4))$.

Proof. By Prop. 4.2.3 of [IR, p. 46], for each $\varepsilon \in\{ \pm 1\}$ and $1 \leqslant s \leqslant r$, we have

$$
\begin{aligned}
& \left|\left\{0 \leqslant x \leqslant p_{s}^{a_{s}}-1: x^{2} \equiv \varepsilon\left(\bmod p_{s}^{a_{s}}\right)\right\}\right| \\
= & \left|\left\{0 \leqslant x \leqslant p_{s}-1: x^{2} \equiv \varepsilon\left(\bmod p_{s}\right)\right\}\right| \\
= & \begin{cases}2 & \text { if } \varepsilon=1 \text { or } p_{s} \equiv 1(\bmod 4), \\
0 & \text { otherwise. }\end{cases}
\end{aligned}
$$

Thus, by applying the Chinese Remainder Theorem we see that

$$
\left|\left\{0 \leqslant x \leqslant m-1: x^{2} \equiv 1 \quad(\bmod m)\right\}\right|=2^{r}
$$

and

$$
\left|\left\{0 \leqslant x \leqslant m-1: x^{2} \equiv \pm 1 \quad(\bmod m)\right\}\right|=2^{(1+\delta) r}
$$


where $\delta$ is 1 or 0 according as whether $p_{s} \equiv 1(\bmod 4)$ for all $s=1, \ldots, r$.

Set $n=(m-1) / 2$ and

$$
S=\{(k, \bar{k}): \operatorname{gcd}(k, m)=1 \& 1 \leqslant k, \bar{k} \leqslant n\} .
$$

Clearly, $(k, \bar{k}) \in S$ if and only if $(\bar{k}, k) \in S$. Note that

$$
k=\bar{k} \in\{1, \ldots, n\} \Longleftrightarrow 1 \leqslant k \leqslant n \& k^{2} \equiv 1(\bmod m) .
$$

Therefore

$$
|S| \equiv\left|\left\{1 \leqslant x<\frac{m}{2}: x^{2} \equiv 1(\bmod m)\right\}\right|=2^{r-1} \equiv \delta_{r, 1} \quad(\bmod 2)
$$

in view of (2.3). This proves (2.1).

In light of (2.4), we have

$$
\begin{aligned}
2 M= & |\{(i, j): 1 \leqslant i, j \leqslant n \& i j \equiv \pm 1(\bmod m)\}| \\
& -\left|\left\{1 \leqslant x \leqslant n: x^{2} \equiv \pm 1(\bmod m)\right\}\right| \\
= & \left|\left\{\left(i, \tau_{m}(i)\right): 1 \leqslant i \leqslant n \& \operatorname{gcd}(i, m)=1\right\}\right|-2^{(1+\delta) r-1} \\
= & \frac{\varphi(m)}{2}-2^{(1+\delta) r-1}=\frac{1}{2} \prod_{s=1}^{r} p_{s}^{a_{s}-1}\left(p_{s}-1\right)-2^{(1+\delta) r-1},
\end{aligned}
$$

which implies that $M$ is odd if and only if $r=1 \&\left(p_{1} \equiv 1\right.$ or $\left.4 a_{1}+3(\bmod 8)\right)$, or $\left(r=2 \& p_{1}+p_{2} \equiv 0(\bmod 4)\right)$. This concludes the proof.

Proof of Theorem 1.1. Set $n=(m-1) / 2$. Clearly $\overline{m-k}=m-\bar{k}$ for all $1 \leqslant k \leqslant m-1$ with $\operatorname{gcd}(k, m)=1$. If $1 \leqslant i<j \leqslant m-1$ with $\operatorname{gcd}(i, m)=$ $\operatorname{gcd}(j, m)=1$, then $m-j<m-i$ and

$$
(\bar{j}-\bar{i})(\overline{m-i}-\overline{m-j})=(\bar{j}-\bar{i})(m-\bar{i}-(m-\bar{j}))=(\bar{j}-\bar{i})^{2}>0 .
$$

If $1 \leqslant i<j \leqslant m-1, \operatorname{gcd}(i, m)=\operatorname{gcd}(j, m)=1$ and $(m-j, m-i)=(i, j)$, then $1 \leqslant i \leqslant n, j=m-i$ and $\bar{j}-\bar{i}=m-2 \bar{i}$. Thus

$\operatorname{sign}\left(\sigma_{m}\right)=(-1)^{|\{1 \leqslant i \leqslant n: \operatorname{gcd}(i, m)=1 \& \bar{i}>n\}|}=(-1)^{\varphi(m) / 2-\delta_{r, 1}}=(-1)^{\delta_{r, 1}\left(p_{1}+1\right) / 2}$ by applying (2.1). This proves (1.1).

Now we turn to show (1.2). For $i, j \in\{1 \leqslant k \leqslant n: \operatorname{gcd}(k, m)=1\}$ with $i<j$, if $i^{*}<j^{*}$ then

$$
\left(j^{*}-i^{*}\right)\left(\left(j^{*}\right)^{*}-\left(i^{*}\right)^{*}\right)=\left(j^{*}-i^{*}\right)(j-i)>0
$$

if $j^{*}<i^{*}$ then

$$
\left(j^{*}-i^{*}\right)\left(\left(i^{*}\right)^{*}-\left(j^{*}\right)^{*}\right)=\left(j^{*}-i^{*}\right)(i-j)>0 ;
$$

if $i=i^{*}$ and $j=j^{*}$ then $j^{*}-i^{*}>0$; if $\left(j^{*}, i^{*}\right)=(i, j)$ then $j^{*}-i^{*}=i-j<0$. In view of this, we see that

$$
\operatorname{sign}\left(\tau_{m}\right)=(-1)^{\left|\left\{1 \leqslant i \leqslant n: \operatorname{gcd}(i, m)=1 \& i<i^{*}\right\}\right|}=(-1)^{M},
$$

where $M$ is given by (2.2). So the second assertion in Theorem 1.1 holds by Lemma 2.1.

The proof of Theorem 1.1 is now complete. 
QUADRATIC RESIDUES AND RELATED PERMUTATIONS AND IDENTITIES 11

Lemma 2.2. Let $p$ be an odd prime, and let $a, b, c \in \mathbb{Z}$ with $a$ or $b$ not divisible by $p$. Then

$$
\sum_{x=0}^{p-1}\left(\frac{a x^{2}+b x+c}{p}\right)= \begin{cases}-\left(\frac{a}{p}\right) & \text { if } p \nmid b^{2}-4 a c \\ (p-1)\left(\frac{a}{p}\right) & \text { if } p \mid b^{2}-4 a c .\end{cases}
$$

Remark 2.1. (2.5) in the case $p \mid a$ is trivial. When $p \nmid a,(2.5)$ is a known result (see, e.g., [BEW, p. 58]).

Lemma 2.3. Let $p$ be any odd prime, and define

$$
r(n):=\mid\left\{(j, k): 1 \leqslant j<k \leqslant \frac{p-1}{2} \text { and } j^{2}+k^{2} \equiv n(\bmod p)\right\} \mid
$$

for $n=0, \ldots, p-1$. Then

$$
r(0)= \begin{cases}(p-1) / 4 & \text { if } p \equiv 1(\bmod 4), \\ 0 & \text { if } p \equiv 3(\bmod 4) .\end{cases}
$$

If $n \in\{1, \ldots, p-1\}$, then

$$
r(n)=\left\lfloor\frac{p+1}{8}\right\rfloor-\frac{1+\left(\frac{2}{p}\right)}{2} \cdot \frac{1+\left(\frac{n}{p}\right)}{2} .
$$

Proof. If $p \equiv 3(\bmod 4)$, then $\left(\frac{-1}{p}\right)=-1$ and hence $r(0)=0$. When $p \equiv 1$ $(\bmod 4)$, we have $q^{2} \equiv-1(\bmod p)$ for some $q \in \mathbb{Z}$, and hence $r(0)=(p-1) / 4$ since

$$
j^{2}+k^{2} \equiv 0 \quad(\bmod p) \Longleftrightarrow j \equiv \pm q k \quad(\bmod p) \Longleftrightarrow k \equiv \mp q j \quad(\bmod p) .
$$

Below we let $n \in\{1, \ldots, p-1\}$. Observe that

$$
\begin{aligned}
2 r(n)+\frac{1+\left(\frac{2 n}{p}\right)}{2}= & 2 r(n)+\left|\left\{1 \leqslant k \leqslant \frac{p-1}{2}: k^{2}+k^{2} \equiv n(\bmod p)\right\}\right| \\
& =\mid\left\{(j, k): 1 \leqslant j, k \leqslant \frac{p-1}{2} \text { and } j^{2}+k^{2} \equiv n(\bmod p)\right\} \mid \\
& =\mid\left\{1 \leqslant x \leqslant p-1:\left(\frac{x}{p}\right)=1 \text { and }\left(\frac{n-x}{p}\right)=1\right\} \mid \\
& =\sum_{x=1} \frac{1+\left(\frac{x}{p}\right)}{2} \cdot \frac{1+\left(\frac{n-x}{p}\right)}{2}-\frac{1+\left(\frac{n}{p}\right)}{2} \cdot \frac{1+\left(\frac{n-n}{p}\right)}{2} \\
= & \frac{p-1}{4}+\frac{1}{4}\left(\sum_{x=0}^{p-1}\left(\frac{x}{p}\right)+\sum_{x=0}^{p-1}\left(\frac{n-x}{p}\right)-\left(\frac{n}{p}\right)\right) \\
& +\frac{1}{4}\left(\frac{-1}{p}\right) \sum_{x=0}^{p-1}\left(\frac{x^{2}-n x}{p}\right)-\frac{1+\left(\frac{n}{p}\right)}{4} \\
= & \frac{p-\left(\frac{-1}{p}\right)}{4}-\frac{1+\left(\frac{n}{p}\right)}{2}
\end{aligned}
$$


with the help of Lemma 2.2. This yields (2.7).

Lemma 2.4. Let $p$ be an odd prime and let $a, b, c \in \mathbb{Z}$ with $a c(a+b+c) \not \equiv 0$ $(\bmod p)$. Write $\Delta=b^{2}-4 a c$. For each $n=0,1 \ldots, p-1$, we have

$$
\begin{aligned}
& \mid\left\{(j, k): 1 \leqslant j<k \leqslant p-1 \text { and } a j^{2}+b j k+c k^{2} \equiv n(\bmod p)\right\} \mid \\
= & \begin{cases}\frac{1}{2}\left(p-3-\left(\frac{\Delta}{p}\right)-\left(\frac{n}{p}\right)\left(\left(1-p+p\left(\frac{\Delta}{p}\right)^{2}\right)\left(\frac{a}{p}\right)+\left(\frac{c}{p}\right)+\left(\frac{a+b+c}{p}\right)\right)\right) & \text { if } n \neq 0, \\
\frac{p-1}{2}\left(1+\left(\frac{\Delta}{p}\right)\right) & \text { if } n=0 .\end{cases}
\end{aligned}
$$

Proof. Let $L$ denote the left-hand side of (2.8). Then

$$
\begin{aligned}
L= & \mid\left\{(j, k): 1 \leqslant j<k \leqslant \frac{p-1}{2} \text { and } a j^{2}+b j k+c k^{2} \equiv n(\bmod p)\right\} \mid \\
& +\mid\left\{(p-j, p-k): 1 \leqslant k \leqslant \frac{p-1}{2}, k<j \leqslant p-1,\right. \\
& \text { and } \left.a(p-j)^{2}+b(p-j)(p-k)+c(p-k)^{2} \equiv n(\bmod p)\right\} \mid \\
= & \mid\left\{(j, k): 1 \leqslant k \leqslant \frac{p-1}{2}, 0 \leqslant j \leqslant p-1, j \neq 0, k,\right. \\
& \text { and } \left.(2 a j+b k)^{2}-\Delta k^{2} \equiv 4 a n(\bmod p)\right\} \mid
\end{aligned}
$$

and hence

$$
\begin{aligned}
L= & \sum_{k=1}^{(p-1) / 2}\left(1+\left(\frac{4 a n+\Delta k^{2}}{p}\right)\right) \\
& -\mid\left\{1 \leqslant k \leqslant \frac{p-1}{2}:(b k)^{2}-\Delta k^{2} \equiv \text { 4an }(\bmod p)\right\} \mid \\
& -\mid\left\{1 \leqslant k \leqslant \frac{p-1}{2}:((2 a+b) k)^{2}-\Delta k^{2} \equiv \text { 4an }(\bmod p)\right\} .
\end{aligned}
$$

In the case $n=0$, this yields

$$
L=\frac{p-1}{2}\left(1+\left(\frac{\Delta}{p}\right)\right) .
$$

When $1 \leqslant n \leqslant p-1$, by the above we have

$$
\begin{aligned}
L= & \sum_{x=1}^{p-1} \frac{1+\left(\frac{x}{p}\right)}{2}\left(1+\left(\frac{\Delta x+4 a n}{p}\right)\right)-\frac{1+\left(\frac{c n}{p}\right)}{2}-\frac{1+\left(\frac{(a+b+c) n}{p}\right)}{2} \\
= & \frac{p-1}{2}+\frac{1}{2} \sum_{x=0}^{p-1}\left(\frac{x}{p}\right)+\frac{1}{2}\left(\sum_{x=0}^{p-1}\left(\frac{\Delta x+4 a n}{p}\right)-\left(\frac{4 a n}{p}\right)\right) \\
& +\frac{1}{2} \sum_{x=0}^{p-1}\left(\frac{\Delta x^{2}+4 a n x}{p}\right)-1-\frac{1}{2}\left(\frac{n}{p}\right)\left(\left(\frac{c}{p}\right)+\left(\frac{a+b+c}{p}\right)\right) \\
= & \frac{p-3}{2}-\frac{1}{2}\left(\frac{\Delta}{p}\right)-\frac{1}{2}\left(\frac{n}{p}\right)\left(\left(1-p \delta_{\left(\frac{\Delta}{p}\right), 0}\right)\left(\frac{a}{p}\right)+\left(\frac{c}{p}\right)+\left(\frac{a+b+c}{p}\right)\right)
\end{aligned}
$$


QUADRATIC RESIDUES AND RELATED PERMUTATIONS AND IDENTITIES 13

with the help of the identity

$$
\sum_{x=0}^{p-1}\left(\frac{\Delta x^{2}+4 a n x}{p}\right)=-\left(\frac{\Delta}{p}\right)
$$

from Lemma 2.2. This proves (2.8).

Lemma 2.5. Let $p$ be an odd prime, and let $a, b, c \in \mathbb{Z}$ with $a+c=0$ and $a b c \not \equiv 0(\bmod p)$. Set $\Delta=b^{2}-4 a c$. Then

$$
(-1)^{\left|\left\{(i, j): 1 \leqslant i, j \leqslant(p-1) / 2 \& p \mid a i^{2}+b i j+c j^{2}\right\}\right|}= \begin{cases}1 & \text { if }\left(\frac{\Delta}{p}\right)=-1 \\ \left(\frac{2}{p}\right) & \text { if }\left(\frac{\Delta}{p}\right)=0 \\ \left(\frac{-1}{p}\right) & \text { if }\left(\frac{\Delta}{p}\right)=1 .\end{cases}
$$

Proof. Define

$$
N=\left|\left\{(i, j): 1 \leqslant i, j \leqslant \frac{p-1}{2} \& p \mid a i^{2}+b i j+c j^{2}\right\}\right| .
$$

Case 1. $\left(\frac{\Delta}{p}\right)=-1$.

In this case,

$$
4 a\left(a i^{2}+b i j+c j^{2}\right)=(2 a i+b j)^{2}-\Delta j^{2} \not \equiv 0 \quad(\bmod p)
$$

for all $i, j=1, \ldots, p-1$. Thus $N=0$ and $(-1)^{N}=1$.

Case 2. $\left(\frac{\Delta}{p}\right)=0$.

In this case, $p$ divides $\Delta=b^{2}+4 a^{2}$, hence $\left(\frac{-1}{p}\right)=1$ and $p \equiv 1(\bmod 4)$. As

$$
\frac{b^{2}}{(2 a)^{2}} \equiv-1 \equiv\left(\frac{p-1}{2} !\right)^{2} \quad(\bmod p)
$$

for some $k=0,1$ we have $x:=(-1)^{k} \frac{p-1}{2} ! \equiv-b /(2 a)(\bmod p)$. Thus

$$
\begin{aligned}
N & =\left|\left\{(i, j): 1 \leqslant i, j \leqslant \frac{p-1}{2} \& i \equiv j x(\bmod p)\right\}\right| \\
& =\frac{p-1}{2}-\left|\left\{1 \leqslant j \leqslant \frac{p-1}{2}:\{j x\}_{p}>\frac{p}{2}\right\}\right|
\end{aligned}
$$

and hence

$$
(-1)^{N}=(-1)^{(p-1) / 2}\left(\frac{x}{p}\right)=\left(\frac{((p-1) / 2) !}{p}\right)=\left(\frac{2}{p}\right)
$$


by using Gauss' Lemma (cf. [IR, p. 52]) and [S19, Lemma 2.3].

Case 3. $\left(\frac{\Delta}{p}\right)=1$.

In this case $\delta^{2} \equiv \Delta$ for some $\delta \in \mathbb{Z}$ with $p \nmid \Delta$. Let $x_{1}$ and $x_{2}$ be integers with $x_{1} \equiv(-b+\delta) /(2 a)(\bmod p)$ and $x_{2} \equiv(-b-\delta) /(2 a)(\bmod p)$. Then $x_{1} \not \equiv x_{2}$ $(\bmod p), x_{1}+x_{2} \equiv-b / a(\bmod p)$ and $x_{1} x_{2} \equiv c / a=-1(\bmod p)$. Thus

$$
\begin{aligned}
N & =\mid\left\{1 \leqslant i, j \leqslant \frac{p-1}{2}: i \equiv j x_{s}(\bmod p) \text { for some } s=1,2\right\} \mid \\
& =\sum_{s=1}^{2}\left(\frac{p-1}{2}-\left|\left\{1 \leqslant j \leqslant \frac{p-1}{2}:\left\{j x_{s}\right\}_{p}>\frac{p}{2}\right\}\right|\right) .
\end{aligned}
$$

Applying Gauss' Lemma we obtain that

$$
(-1)^{N}=(-1)^{p-1} \prod_{s=1}^{2}\left(\frac{x_{s}}{p}\right)=\left(\frac{x_{1} x_{2}}{p}\right)=\left(\frac{-1}{p}\right) .
$$

In view of the above, we have completed the proof of Lemma 2.5.

Lemma 2.6. For any odd prime $p$, we have

$$
\prod_{1 \leqslant i<j \leqslant p-1}(j-i) \equiv-\left(\frac{2}{p}\right) \frac{p-1}{2} ! \quad(\bmod p) .
$$

Proof. Clearly $\left(\begin{array}{c}p-1 \\ k\end{array}\right) \equiv(-1)^{k}(\bmod p)$ for all $k=0, \ldots, p-1$. Also, $(p-1) ! \equiv$ $-1(\bmod p)$ by Wilson's theorem. Thus

$$
\begin{aligned}
\prod_{1 \leqslant i<j \leqslant p-1}(j-i) & =\prod_{j=2}^{p-1}(j-1) !=\prod_{k=1}^{p-2} k ! \\
& =\frac{p-1}{2} ! \prod_{0<k<(p-1) / 2} \frac{(p-1) !}{\left(\begin{array}{c}
p-1 \\
k
\end{array}\right)} \equiv \frac{p-1}{2} ! \prod_{0<k<(p-1) / 2}(-1)^{k-1} \\
& \equiv \frac{p-1}{2} !(-1)^{(p-3)(p-5) / 8}=\frac{p-1}{2} !(-1)^{\left(p^{2}-9\right) / 8} \\
& \equiv-\left(\frac{2}{p}\right) \frac{p-1}{2} !(\bmod p) .
\end{aligned}
$$

This proves $(2.10)$.

Lemma 2.7. Let $p$ be an odd prime, and let $a, b \in \mathbb{Z}$ with $a \not \equiv 0,1(\bmod p)$. Then

$$
\left|\left\{x \in\{0,1 \ldots, p-1\}:\{a x+b\}_{p}>x\right\}\right|=\frac{p-1}{2} .
$$


QUADRATIC RESIDUES AND RELATED PERMUTATIONS AND IDENTITIES 15

Proof. For $x \in\{0, \ldots, p-1\}$, obviously

$$
\{a x+b+1\}_{p}>x \Longleftrightarrow p-1>\{a x+b\}_{p} \geqslant x .
$$

As $a \not \equiv 0,1(\bmod p)$, we have

$\left|\left\{x \in\{0, \ldots, p-1\}:\{a x+b\}_{p}=x\right\}\right|=1=\left|\left\{x \in\{0, \ldots, p-1\}:\{a x+b\}_{p}=p-1\right\}\right|$.

Thus

$\left|\left\{x \in\{0, \ldots, p-1\}:\{a x+b+1\}_{p}>x\right\}\right|=\left|\left\{x \in\{0, \ldots, p-1\}:\{a x+b\}_{p}>x\right\}\right|$.

In view of the above, it suffices to prove (2.11) for $b=0$. For $x=1, \ldots, p-1$, clearly $\{a x\}_{p} \neq x($ since $a \not \equiv 1(\bmod p))$, and also

$$
\{a x\}_{p}>x \Longleftrightarrow p-\{a x\}_{p}<p-x \Longleftrightarrow\{a(p-x)\}_{p}<p-x .
$$

So (2.11) holds for $b=0$. This concludes the proof.

Proof of Theorem 1.2. (i) Let $r(n)$ be as in Lemma 2.3. Then

$$
\begin{aligned}
\prod_{\substack{1 \leqslant i<j \leqslant(p-1) / 2 \\
p \nmid i^{2}+j^{2}}}\left(i^{2}+j^{2}\right) & \equiv \prod_{n=1}^{p-1} n^{r(n)}=((p-1) !)^{\lfloor(p+1) / 8\rfloor} \prod_{\substack{n=1 \\
\left(\frac{n}{p}\right)=1}}^{p-1} n^{-\left(1+\left(\frac{2}{p}\right)\right) / 2} \\
& \equiv(-1)^{\lfloor(p+1) / 8\rfloor} \prod_{k=1}^{(p-1) / 2}\left(k^{2}\right)^{-\left(1+\left(\frac{2}{p}\right)\right) / 2} \\
& \equiv(-1)^{\lfloor(p+1) / 8\rfloor}\left((-1)^{(p+1) / 2}\right)^{\left(1+\left(\frac{2}{p}\right)\right) / 2}(\bmod p)
\end{aligned}
$$

with the help of (1.3). This yields $(1.7)$ if $p \equiv 1(\bmod 4)$. It also proves $(1.6)$ in the case $p \equiv 3(\bmod 4)$.

(ii) If $p \mid \Delta$, then by Lemma 2.4 and (1.3) we have

$$
\begin{aligned}
& \prod_{\substack{1 \leqslant i<j \leqslant(p-1) / 2 \\
p \nmid a i^{2}+b i j+c j^{2}}}\left(a i^{2}+b i j+c j^{2}\right) \\
\equiv & \prod_{n=1}^{p-1} n^{\frac{p-3}{2}+\frac{1-p}{2}\left(\frac{a}{p}\right)+\frac{1}{2}\left(\left(\frac{c}{p}\right)+\left(\frac{a+b+c}{p}\right)\right)-\frac{1}{2}\left(1+\left(\frac{n}{p}\right)\right)\left((1-p)\left(\frac{a}{p}\right)+\left(\frac{c}{p}\right)+\left(\frac{a+b+c}{p}\right)\right)} \\
\equiv & (-1)^{\frac{p-3}{2}+\frac{1-p}{2}\left(\frac{a}{p}\right)+\frac{1}{2}\left(\left(\frac{c}{p}\right)+\left(\frac{a+b+c}{p}\right)\right)} \prod_{k=1}^{(p-1) / 2}\left(k^{2}\right)^{-\left((1-p)\left(\frac{a}{p}\right)+\left(\frac{c}{p}\right)+\left(\frac{a+b+c}{p}\right)\right)} \\
\equiv & (-1)^{\frac{1}{2}\left(\left(\frac{c}{p}\right)+\left(\frac{a+b+c}{p}\right)\right)-1}\left((-1)^{(p+1) / 2}\right)(1-p)\left(\frac{a}{p}\right)+\left(\frac{c}{p}\right)+\left(\frac{a+b+c}{p}\right) \\
= & (-1)^{\frac{1}{2}\left(\left(\frac{c}{p}\right)+\left(\frac{a+b+c}{p}\right)\right)-1}=\left(\frac{c(a+b+c)}{p}\right)=\left(\frac{a(a+b+c)}{p}\right)(\bmod p)
\end{aligned}
$$


since $4 a c \equiv b^{2}(\bmod p)$. Similarly, when $p \nmid \Delta$, by Lemma 2.4 and (1.3) we have

$$
\begin{aligned}
& \prod_{\substack{1 \leqslant i<j \leqslant(p-1) / 2 \\
p \nmid a i^{2}+b i j+c j^{2}}}\left(a i^{2}+b i j+c j^{2}\right) \\
\equiv & \prod_{n=1}^{p-1} n^{\frac{p-3}{2}+\frac{1}{2}\left(\left(\frac{a}{p}\right)+\left(\frac{c}{p}\right)+\left(\frac{a+b+c}{p}\right)-\left(\frac{\Delta}{p}\right)\right)-\frac{1}{2}\left(1+\left(\frac{n}{p}\right)\right)\left(\left(\frac{a}{p}\right)+\left(\frac{c}{p}\right)+\left(\frac{a+b+c}{p}\right)\right)} \\
\equiv & (-1)^{\frac{p-3}{2}+\frac{1}{2}\left(\left(\frac{a}{p}\right)+\left(\frac{c}{p}\right)+\left(\frac{a+b+c}{p}\right)-\left(\frac{\Delta}{p}\right)\right)} \prod_{k=1}^{(p-1) / 2}\left(k^{2}\right)^{-\left(\left(\frac{a}{p}\right)+\left(\frac{c}{p}\right)+\left(\frac{a+b+c}{p}\right)\right)} \\
\equiv & (-1)^{\frac{p-3}{2}+\frac{1}{2}\left(\left(\frac{a}{p}\right)+\left(\frac{c}{p}\right)+\left(\frac{a+b+c}{p}\right)-\left(\frac{\Delta}{p}\right)\right)}\left((-1)^{(p+1) / 2}\right)^{\left(\frac{a}{p}\right)+\left(\frac{c}{p}\right)+\left(\frac{a+b+c}{p}\right)}(\bmod p)
\end{aligned}
$$

and hence

$$
\begin{aligned}
\prod_{\substack{1 \leqslant i<j \leqslant(p-1) / 2 \\
p \nmid a i^{2}+b i j+c j^{2}}}\left(a i^{2}+b i j+c j^{2}\right) & \equiv(-1)^{\frac{1}{2}\left(\left(\frac{a}{p}\right)+\left(\frac{c}{p}\right)\right)}(-1)^{\frac{1}{2}\left(\left(\frac{a+b+c}{p}\right)-\left(\frac{\Delta}{p}\right)\right)} \\
& =-\left(\frac{a c}{p}\right)\left(\frac{(a+b+c) \Delta}{p}\right)(\bmod p) .
\end{aligned}
$$

This proves (1.8).

Now assume that $a+c=0$. We deduce (1.9) from (1.8). Observe that

$$
\begin{aligned}
& \prod_{1 \leqslant i<j \leqslant p-1}\left(a i^{2}+b i j+c j^{2}\right) \\
& p \nmid a i^{2}+b i j+c j^{2} \\
& =\prod_{\substack{i, j=1 \\
p \nmid a i^{2}-b i j+c j^{2}}}^{(p-1) / 2}\left(a i^{2}+b i(p-j)+c(p-j)^{2}\right) \times \prod_{\substack{1 \leqslant i<j \leqslant(p-1) / 2 \\
p \nmid a i^{2}+b i j+c j^{2}}}\left(a i^{2}+b i j+c j^{2}\right) \\
& \times \prod_{\substack{1 \leqslant j<i \leqslant(p-1) / 2 \\
p \nmid a i^{2}+b i j+c j^{2}}}\left(a(p-i)^{2}+b(p-i)(p-j)+c(p-j)^{2}\right) \\
& \equiv \prod_{\substack{i, j=1 \\
p \nmid a i^{2}-b i j+c j^{2}}}^{(p-1) / 2}\left(a i^{2}-b i j+c j^{2}\right) \times \prod_{\substack{i, j=1 \\
p \nmid a i^{2}+b i j+c j^{2}}}^{(p-1) / 2}\left(a i^{2}+b i j+c j^{2}\right) / \prod_{i=1}^{(p-1) / 2}\left(a i^{2}+b i^{2}+c i^{2}\right) \\
& \equiv \frac{(-1)^{m}}{((p-1) / 2) !^{2}}\left(\frac{a+b+c}{p}\right) \prod_{\substack{i, j=1 \\
p \nmid c i^{2}+b i j+a j^{2}}}^{(p-1) / 2}\left(c i^{2}+b i j+a j^{2}\right) \times \prod_{\substack{i, j=1 \\
p \nmid a i^{2}+b i j+c j^{2}}}^{(p-1) / 2}\left(a i^{2}+b i j+c j^{2}\right) \\
& =\frac{(-1)^{m}}{((p-1) / 2) !)^{2}}\left(\frac{a+b+c}{p}\right) \prod_{\substack{i, j=1 \\
p \nmid a i^{2}+b i j+c j^{2}}}^{(p-1) / 2}\left(a i^{2}+b i j+c j^{2}\right)^{2}(\bmod p),
\end{aligned}
$$


QUADRATIC RESIDUES AND RELATED PERMUTATIONS AND IDENTITIES 17

where

$$
\begin{aligned}
m & =\mid\left\{(i, j): 1 \leqslant i, j \leqslant \frac{p-1}{2} \text { and } p \nmid a i^{2}-b i j+c j^{2}\right\} \mid \\
& =\mid\left\{(i, j): 1 \leqslant i, j \leqslant \frac{p-1}{2} \text { and } p \nmid c i^{2}+b i j+a j^{2}\right\} \mid .
\end{aligned}
$$

Combining this with (1.8) and noting $a c=-a^{2}$, we see that

$$
\prod_{\substack{i, j=1 \\ p \nmid a i^{2}+b i j+c j^{2}}}^{(p-1) / 2}\left(a i^{2}+b i j+c j^{2}\right)^{2} \equiv(-1)^{m}\left(\frac{p-1}{2} !\right)^{2} \times \begin{cases}\left(\frac{a}{p}\right)(\bmod p) & \text { if } p \mid \Delta, \\ -\left(\frac{-\Delta}{p}\right)(\bmod p) & \text { if } p \nmid \Delta .\end{cases}
$$

By Lemma 2.5,

$$
(-1)^{((p-1) / 2)^{2}-m}= \begin{cases}1 & \text { if }\left(\frac{\Delta}{p}\right)=-1, \\ \left(\frac{2}{p}\right) & \text { if }\left(\frac{\Delta}{p}\right)=0 \\ \left(\frac{-1}{p}\right) & \text { if }\left(\frac{\Delta}{p}\right)=1 .\end{cases}
$$

If $p$ divides $\Delta=b^{2}+4 a^{2}$, then $\left(\frac{-1}{p}\right)=1,(-1)^{m}=\left(\frac{2}{p}\right),\left(\frac{b}{2 a}\right)^{2} \equiv-1 \equiv\left(\frac{p-1}{2} !\right)^{2}$ $(\bmod p)$ and

$$
\left(\frac{b}{p}\right)=\left(\frac{ \pm 2 \times((p-1) / 2) !}{p}\right)\left(\frac{a}{p}\right)=\left(\frac{a}{p}\right)
$$

with the help of [S19, Lemma 2.3]. Thus, in view of (2.12) and (1.3), we have (1.9) if $p \mid \Delta$. When $\left(\frac{\Delta}{p}\right)=-1$, we have

$$
-(-1)^{m}\left(\frac{-\Delta}{p}\right)=(-1)^{m}\left(\frac{-1}{p}\right)=1
$$

and hence (1.9) holds in view of (2.12). If $\left(\frac{\Delta}{p}\right)=1$, then

$$
-(-1)^{m}\left(\frac{-\Delta}{p}\right)=-(-1)^{m}\left(\frac{-1}{p}\right)=-\left(\frac{-1}{p}\right) \equiv \frac{1}{((p-1) / 2) !^{2}} \quad(\bmod p)
$$

and hence (1.9) follows from (2.12).

(iii) If $a \equiv c(\bmod p)$, then $b \equiv-a-c \equiv-2 a(\bmod p)$ and hence

$$
\begin{aligned}
& \prod_{\substack{1 \leqslant i<j \leqslant p-1 \\
p \nmid a i^{2}+b i j+c j^{2}}}\left(a i^{2}+b i j+c j^{2}\right) \\
\equiv & \prod_{1 \leqslant i<j \leqslant p-1} a(j-i)^{2}=a^{\frac{p-1}{2}(p-2)} \prod_{1 \leqslant i<j \leqslant p-1}(j-i)^{2} \\
\equiv & \left(\frac{a}{p}\right)\left(\frac{p-1}{2} !\right)^{2} \equiv(-1)^{(p+1) / 2}\left(\frac{a}{p}\right)(\bmod p)
\end{aligned}
$$


with the help of (2.10) and (1.3).

Now assume that $a \not \equiv c(\bmod p)$. For $1 \leqslant i<j \leqslant p-1$, clearly

$$
a i^{2}+b i j+c j^{2} \equiv(i-j)(a i-c j) \equiv c(j-i)\left(j-\frac{a}{c} i\right) \quad(\bmod p) .
$$

Note that

$$
\begin{aligned}
\prod_{\substack{1 \leqslant i<j \leqslant p-1 \\
p \nmid a i-c j}}\left(j-\frac{a}{c} i\right) & \equiv \prod_{r=1}^{p-1} r^{\left|\left\{(i, j): 1 \leqslant i<j \leqslant p-1 \& j-\frac{a}{c} i \equiv r(\bmod p)\right\}\right|} \\
& \equiv \prod_{r=1}^{p-1} r^{\left|\left\{1 \leqslant i \leqslant p-1:\left\{r+\frac{a}{c} i\right\}_{p}>i\right\}\right|} \\
& \equiv(p-1) !^{(p-1) / 2-1} \equiv(-1)^{(p+1) / 2}(\bmod p)
\end{aligned}
$$

with the help of Lemma 2.7 and Wilson's theorem. Also,

$$
\begin{aligned}
\prod_{\substack{1 \leqslant i<j \leqslant p-1 \\
p \mid a i-c j}} c(j-i) & \equiv \prod_{\substack{i=1 \\
\left\{\frac{a}{c} i\right\}_{p}>i}}^{p-1} c\left(\frac{a}{c} i-i\right) \\
& \equiv \prod_{\substack{i=1 \\
\left\{\frac{a}{c} i\right\}_{p}>i}}^{(p-1) / 2}(a-c) i \times \prod_{\substack{i=1 \\
c}}^{(p-i)\}_{p}>p-i}(a-c)(p-i) \\
& \equiv \prod_{\substack{(p-1) / 2 \\
i=1}}^{(p-1) / 2}(a-c) i \times(-1)^{\left|\left\{1 \leqslant i \leqslant \frac{p-1}{2}:\left\{\frac{a}{c} i\right\}_{p}<i\right\}\right|} \\
& \equiv\left(\frac{a-c}{p}\right) \frac{p-1}{2} !(-1)^{(p-1) / 2-N_{p}(a / c)}(\bmod p)
\end{aligned}
$$

and hence

$$
\begin{aligned}
\prod_{\substack{1 \leqslant i<j \leqslant p-1 \\
p \nmid a i c j}} c(j-i) & \equiv \frac{\prod_{1 \leqslant i<j \leqslant p-1} c(j-i)}{\left(\frac{a-c}{p}\right) \frac{p-1}{2} !(-1)^{(p-1) / 2-N_{p}(a / c)}} \\
& \equiv \frac{-c^{(p-1)(p-2) / 2}\left(\frac{2}{p}\right) \frac{p-1}{2} !}{\left(\frac{a-c}{p}\right) \frac{p-1}{2} !(-1)^{(p-1) / 2-N_{p}(a / c)}} \\
& \equiv\left(\frac{2 c(a-c)}{p}\right)(-1)^{(p+1) / 2+N_{p}(a / c)}(\bmod p)
\end{aligned}
$$

with the help of (2.10). Therefore

$$
\begin{aligned}
\prod_{\substack{1 \leqslant i<j \leqslant p-1 \\
p \nmid a i^{2}+b i j+c j^{2}}}\left(a i^{2}+b i j+c j^{2}\right) & \equiv \prod_{\substack{1 \leqslant i<j \leqslant p-1 \\
p \nmid a i-c j}} c(j-i) \times \prod_{\substack{1 \leqslant i<j \leqslant p-1 \\
p \nmid a i-c j}}\left(j-\frac{a}{c} i\right) \\
& \equiv\left(\frac{2 c(a-c)}{p}\right)(-1)^{N_{p}(a / c)}(\bmod p) .
\end{aligned}
$$


This proves part (iii) of Theorem 1.2.

(iv) In the spirit of our proof of Theorem 1.2(iii), we may show Theorem 1.2(iv). To illustrate this, here we handle the case $p \mid a$ and $p \nmid b c(b+c)$ in details. Note that

$$
\prod_{\substack{1 \leqslant i<j \leqslant p-1 \\ p \nmid a i^{2}+b i j+c j^{2}}}\left(a i^{2}+b i j+c j^{2}\right) \equiv \prod_{\substack{1 \leqslant i<j \leqslant p-1 \\ p \nmid b i+c j}} c j \times \prod_{\substack{1 \leqslant i<j \leqslant p-1 \\ p \nmid b i+c j}}\left(j+\frac{b}{c} i\right) \quad(\bmod p) .
$$

Similar to the second paragraph in (iii), we have

$$
\prod_{\substack{1 \leqslant i<j \leqslant p-1 \\ p \nmid b i+c j}}\left(j+\frac{b}{c} i\right) \equiv(-1)^{(p+1) / 2} \quad(\bmod p) .
$$

Observe that

$$
\begin{aligned}
\prod_{\substack{1 \leqslant i<j \leqslant p-1 \\
p \mid b i+c j}} c j & =\prod_{\substack{j=1 \\
\left\{-\frac{c}{b} j\right\}_{p}<j}}^{(p-1) / 2} c j \times \prod_{\substack{j=1 \\
\left\{-\frac{c}{b}(p-j)\right\}_{p}<p-j}}^{(p-1) / 2} c(p-j) \\
& \equiv \prod_{j=1}^{(p-1) / 2} c j \times(-1)^{\left|\left\{1 \leqslant j \leqslant \frac{p-1}{2}:\left\{-\frac{c}{b} j\right\}_{p}>j\right\}\right|} \\
& \equiv\left(\frac{c}{p}\right) \frac{p-1}{2} !(-1)^{N_{p}(-c / b)}(\bmod p)
\end{aligned}
$$

and hence

$$
\begin{aligned}
\prod_{\substack{1 \leqslant i<j \leqslant p-1 \\
p \nmid b i+c j}} c j & \equiv \frac{\prod_{j=1}^{(p-1) / 2}(c j)^{j-1}(c(p-j))^{p-j-1}}{\left(\frac{c}{p}\right) \frac{p-1}{2} !(-1)^{N_{p}(-c / b)}} \\
& \equiv \prod_{j=1}^{(p-1) / 2} \frac{(-1)^{j}(c j)^{p-1}}{c j} \times \frac{\left(\frac{c}{p}\right)(-1)^{N_{p}(-c / b)}}{\frac{p-1}{2} !} \\
& \equiv(-1)^{\left(p^{2}-1\right) / 8+N_{p}(-c / b)}\left(\frac{p-1}{2} !\right)^{-2} \\
& \equiv(-1)^{(p+1) / 2+N_{p}(-c / b)}\left(\frac{2}{p}\right)(\bmod p)
\end{aligned}
$$

with the help of (1.3). Combining this with (2.13) and (2.14), we obtain that

$$
\prod_{\substack{1 \leqslant i<j \leqslant p-1 \\ p \nmid a i^{2}+b i j+c j^{2}}}\left(a i^{2}+b i j+c j^{2}\right) \equiv(-1)^{N_{p}(-c / b)}\left(\frac{2}{p}\right) \quad(\bmod p) .
$$

This ends our proof. 


\section{Proof of Theorem 1.3}

To prove Theorem 1.3, we need some known results.

Lemma 3.1. Let $p$ be an odd prime, and let $\zeta=e^{2 \pi i / p}$.

(i) For any $a \in \mathbb{Z}$ with $p \nmid a$, we have

$$
\prod_{n=1}^{p-1}\left(1-\zeta^{a n}\right)=p
$$

and

$$
\sum_{x=0}^{p-1} \zeta^{a x^{2}}=\left(\frac{a}{p}\right) \sqrt{(-1)^{(p-1) / 2} p}
$$

(ii) If $p \equiv 1(\bmod 4)$, then

$$
\prod_{n=1}^{p-1}\left(1-\zeta^{n}\right)^{\left(\frac{n}{p}\right)}=\varepsilon_{p}^{-2 h(p)}
$$

(iii) When $p \equiv 3(\bmod 4)$, we have

$$
p h(-p)=-\sum_{k=1}^{p-1} k\left(\frac{k}{p}\right),
$$

and also

$$
\left|\left\{1 \leqslant k \leqslant \frac{p-1}{2}:\left(\frac{k}{p}\right)=-1\right\}\right| \equiv \frac{h(-p)+1}{2} \quad(\bmod 2)
$$

provided $p>3$.

Remark 3.1. This lemma is well known. For any $a \in \mathbb{Z}$ with $p \nmid a$, we have (3.1) since

$$
\prod_{n=1}^{p-1}\left(1-\zeta^{a n}\right)=\prod_{k=1}^{p-1}\left(1-\zeta^{k}\right)=\lim _{x \rightarrow 1} \frac{x^{p}-1}{x-1}=p
$$

and we have (3.2) by Gauss' evaluation of quadratic Gauss sums (cf. [IR, pp. 7075]). Part (ii) and the first assertion in part (iii) are Dirichlet's class number formula. The second assertion in part (iii) was pointed out by Mordell [M61]. 
QUADRATIC RESIDUES AND RELATED PERMUTATIONS AND IDENTITIES 21

Lemma 3.2. Let $p$ be an odd prime and let $n \in\{1, \ldots, p-1\}$. Then

$$
\begin{aligned}
& \mid\left\{(j, k): 1 \leqslant j, k \leqslant \frac{p-1}{2} \text { and } j^{2}-k^{2} \equiv n(\bmod p)\right\} \mid \\
= & \left\lfloor\frac{p-1}{4}\right\rfloor- \begin{cases}1 & \text { if } p \equiv 1(\bmod 4) \text { and }\left(\frac{n}{p}\right)=1, \\
0 & \text { otherwise. }\end{cases}
\end{aligned}
$$

Proof. Let $L$ denote the left-hand side of (3.6). Then

$$
\begin{aligned}
L= & \mid\left\{1 \leqslant x \leqslant p-1:\left(\frac{x}{p}\right)=1 \text { and }\left(\frac{n+x}{p}\right)=1\right\} \mid \\
= & \sum_{x=1}^{p-1} \frac{\left(\frac{x}{p}\right)+1}{2} \cdot \frac{\left(\frac{x+n}{p}\right)+1}{2}-\frac{\left(\frac{p-n}{p}\right)+1}{2} \cdot \frac{\left(\frac{p-n+n}{p}\right)+1}{2} \\
= & \frac{p-1}{4}+\frac{1}{4} \sum_{x=0}^{p-1}\left(\frac{x}{p}\right)+\frac{1}{4}\left(\sum_{x=0}^{p-1}\left(\frac{x+n}{p}\right)-\left(\frac{n}{p}\right)\right) \\
& +\frac{1}{4} \sum_{x=0}^{p-1}\left(\frac{x(x+n)}{p}\right)-\frac{\left(\frac{-n}{p}\right)+1}{4} \\
= & \frac{p-3-\left(\frac{n}{p}\right)-\left(\frac{-n}{p}\right)}{4}
\end{aligned}
$$

with the help of Lemma 2.2. This yields (3.6).

Proof of Theorem 1.3. Let $\varphi_{a}$ be the element of the $\operatorname{Galois} \operatorname{group} \operatorname{Gal}(\mathbb{Q}(\zeta) / \mathbb{Q})$ with $\varphi_{a}(\zeta)=\zeta^{a}$. In view of $(3.2)$,

$$
\varphi_{a}\left(\sqrt{(-1)^{(p-1) / 2} p}\right)=\varphi_{a}\left(\sum_{x=0}^{p-1} \zeta^{x^{2}}\right)=\sum_{x=0}^{p-1} \zeta^{a x^{2}}=\left(\frac{a}{p}\right) \sqrt{(-1)^{(p-1) / 2} p}
$$

(i) We first handle the case $p \equiv 1(\bmod 4)$. Combining (3.1) and (3.3), we get

$$
\prod_{\substack{n=1 \\\left(\frac{n}{p}\right)=1}}^{p-1}\left(1-\zeta^{n}\right)^{2}=\prod_{n=1}^{p-1}\left(1-\zeta^{n}\right)^{1+\left(\frac{n}{p}\right)}=p \varepsilon_{p}^{-2 h(p)}
$$

Note that

$$
\prod_{\substack{n=1 \\\left(\frac{n}{p}\right)=1}}^{p-1}\left(1-\zeta^{n}\right)=\prod_{\substack{n=1 \\\left(\frac{n}{p}\right)=1}}^{(p-1) / 2}\left(1-\zeta^{n}\right)\left(1-\zeta^{p-n}\right)=\prod_{\substack{n=1 \\\left(\frac{n}{p}\right)=1}}^{(p-1) / 2}\left|1-\zeta^{n}\right|^{2}>0
$$


Therefore

$$
\prod_{k=1}^{(p-1) / 2}\left(1-\zeta^{k^{2}}\right)=\prod_{\substack{n=1 \\\left(\frac{n}{p}\right)=1}}^{p-1}\left(1-\zeta^{n}\right)=\sqrt{p} \varepsilon_{p}^{-h(p)}
$$

This proves (1.12) for $a=1$.

Write $\varepsilon_{p}=u_{p}+v_{p} \sqrt{p}$ with $u_{p}, v_{p} \in \mathbb{Q}$. In view of $(3.7)$,

$$
\varphi_{a}\left(\varepsilon_{p}\right)=u_{p}+\left(\frac{a}{p}\right) v_{p} \sqrt{p}=\frac{N\left(\varepsilon_{p}\right)}{u_{p}-\left(\frac{a}{p}\right) v_{p} \sqrt{p}}= \begin{cases}\varepsilon_{p} & \text { if }\left(\frac{a}{p}\right)=1 \\ N\left(\varepsilon_{p}\right) \varepsilon_{p}^{-1} & \text { if }\left(\frac{a}{p}\right)=-1\end{cases}
$$

where $N\left(\varepsilon_{p}\right)$ is the norm of $\varepsilon_{p}$ with respect to the field extension $\mathbb{Q}(\sqrt{p}) / \mathbb{Q}$. Thus, by using (3.7) and (3.8) we obtain

$$
\begin{aligned}
\prod_{k=1}^{(p-1) / 2}\left(1-\zeta^{a k^{2}}\right) & =\varphi_{a}\left(\prod_{k=1}^{(p-1) / 2}\left(1-\zeta^{k^{2}}\right)\right)=\varphi_{a}\left(\sqrt{p} \varepsilon_{p}^{-h(p)}\right) \\
& = \begin{cases}\sqrt{p} \varepsilon_{p}^{-h(p)} & \text { if }\left(\frac{a}{p}\right)=1, \\
-\sqrt{p} N\left(\varepsilon_{p}\right)^{-h(p)} \varepsilon_{p}^{h(p)} & \text { if }\left(\frac{a}{p}\right)=-1 .\end{cases}
\end{aligned}
$$

This proves (1.12) since $N\left(\varepsilon_{p}\right)^{h(p)}=-1$ (cf. [Co, p. 185 and p. 187]).

Now we consider the case $p \equiv 3(\bmod 4)$. In view of $(3.4)$,

$$
\begin{aligned}
p h(-p) & =-\sum_{r=1}^{(p-1) / 2}\left(r\left(\frac{r}{p}\right)+(p-r)\left(\frac{p-r}{p}\right)\right) \\
& =-2 \sum_{r=1}^{(p-1) / 2} r\left(\frac{r}{p}\right)+p \sum_{r=1}^{(p-1) / 2}\left(\frac{r}{p}\right)
\end{aligned}
$$

and hence $p \mid \sum_{r=1}^{(p-1) / 2} r\left(\frac{r}{p}\right)$. Let $N=\left|\left\{1 \leqslant r \leqslant(p-1) / 2:\left(\frac{r}{p}\right)=-1\right\}\right|$. 
QUADRATIC RESIDUES AND RELATED PERMUTATIONS AND IDENTITIES 23

Observe that

$$
\begin{aligned}
\prod_{k=1}^{(p-1) / 2}\left(1-\zeta^{k^{2}}\right) & =\prod_{\substack{k=1 \\
(p-1) / 2}}^{(p-1) / 2}\left(1-\zeta^{(2 k)^{2}}\right) \\
& =\prod_{\substack{r=1 \\
\left(\frac{r}{p}\right)=1}}^{(p-1) / 2}\left(1-\zeta^{4 r}\right) \times \prod_{\substack{r=1 \\
\left(\frac{r}{p}\right)=-1}}^{(p-1) / 2}\left(1-\zeta^{4(p-r)}\right) \\
& =\prod_{\substack{r=1 \\
\left(\frac{r}{p}\right)=1}}^{(p-1) / 2}\left(1-\zeta^{4 r}\right) \times \prod_{\substack{r=1 \\
\left(\frac{r}{p}\right)=-1}}^{\left(\zeta^{4 r}-1\right.} \zeta^{4 r} \\
& =(-1)^{N} \zeta^{-4 \sum_{0<r<p / 2,\left(\frac{r}{p}\right)=-1}} \prod_{r=1}^{(p-1) / 2} \zeta^{2 r}\left(\zeta^{-2 r}-\zeta^{2 r}\right) \\
& =(-1)^{N} \zeta^{\sum_{r=1}^{(p-1) / 2} 2 r\left(\frac{r}{p}\right)} \prod_{r=1}^{(p-1) / 2}\left(\zeta^{-2 r}-\zeta^{2 r}\right) \\
& =(-1)^{N} \prod_{r=1}^{(p-1) / 2}\left(\zeta^{-2 r}-\zeta^{2 r}\right)
\end{aligned}
$$

and hence

$$
(-1)^{N} \prod_{k=1}^{(p-1) / 2}\left(1-\zeta^{k^{2}}\right)=(-1)^{(p-1) / 2} \prod_{r=1}^{(p-1) / 2}\left(\zeta^{2 r}-\zeta^{-2 r}\right)
$$

By Prop. 6.4.3 of [IR, p. 74],

$$
\prod_{r=1}^{(p-1) / 2}\left(\zeta^{2 r-1}-\zeta^{-(2 r-1)}\right)=\sqrt{p} i
$$

Thus

$$
\sqrt{p} i \prod_{r=1}^{(p-1) / 2}\left(\zeta^{2 r}-\zeta^{-2 r}\right)=\prod_{k=1}^{p-1}\left(\zeta^{k}-\zeta^{-k}\right)=\zeta^{\sum_{k=1}^{p-1} k} \prod_{k=1}^{p-1}\left(1-\zeta^{-2 k}\right)=p
$$

with the help of (3.1). Combining this with (3.9) we obtain

$$
(-1)^{N} \prod_{k=1}^{(p-1) / 2}\left(1-\zeta^{k^{2}}\right)=\sqrt{p} i
$$


Therefore, by using (3.7) we get

$$
(-1)^{N} \prod_{k=1}^{(p-1) / 2}\left(1-\zeta^{a k^{2}}\right)=\varphi_{a}(\sqrt{p} i)=\left(\frac{a}{p}\right) \sqrt{p} i .
$$

This yields $(1.13)$ since $N \equiv(h(-p)+1) / 2(\bmod 2)$ by $(3.5)$.

(ii) Observe that

$$
\begin{aligned}
& \prod_{1 \leqslant j<k \leqslant(p-1) / 2}\left(\zeta^{a j^{2}}-\zeta^{a k^{2}}\right)^{2} \\
= & (-1)^{\left(\begin{array}{c}
(p-1) / 2 \\
2
\end{array}\right)} \prod_{1 \leqslant j<k \leqslant(p-1) / 2}\left(\zeta^{a j^{2}}-\zeta^{a k^{2}}\right)\left(\zeta^{a k^{2}}-\zeta^{a j^{2}}\right) \\
= & (-1)^{\left(\begin{array}{c}
(p-1) / 2 \\
2
\end{array}\right)} \prod_{k=1}^{(p-1) / 2} \prod_{\substack{j=1 \\
j \neq k}}^{(p-1) / 2}\left(\zeta^{a k^{2}}-\zeta^{a j^{2}}\right) \\
= & (-1)^{\left(\begin{array}{c}
(p-1) / 2 \\
2
\end{array}\right)} \prod_{k=1}^{(p-1) / 2}\left(\zeta^{a k^{2}}\right)^{(p-3) / 2} \times \prod_{\substack{k=1 \\
j=1}}^{(p-1) / 2} \prod_{\substack{j \neq k \\
j \neq k}}^{(p-1) / 2}\left(1-\zeta^{a\left(j^{2}-k^{2}\right)}\right) \\
= & (-1)^{(p-1)(p-3) / 8} \zeta^{\frac{p-3}{2} \sum_{k=1}^{(p-1) / 2} a k^{2}} \prod_{\substack{j, k=1 \\
j \neq k}}^{(p-1) / 2}\left(1-\zeta^{a\left(j^{2}-k^{2}\right)}\right) .
\end{aligned}
$$

Clearly,

$$
\sum_{k=1}^{(p-1) / 2} k^{2}=\frac{p^{2}-1}{24} p \equiv 0 \quad(\bmod p) .
$$

So, with the help of Lemma 3.2, from the above we obtain

$$
\begin{aligned}
\prod_{1 \leqslant j<k \leqslant(p-1) / 2}\left(\zeta^{a j^{2}}-\zeta^{a k^{2}}\right)^{2}= & (-1)^{(p-1)(p-3) / 8} \prod_{n=1}^{p-1}\left(1-\zeta^{a n}\right)^{\lfloor(p-1) / 4\rfloor} \\
& \times \begin{cases}\prod_{0<n<p,\left(\frac{n}{p}\right)=1}\left(1-\zeta^{a n}\right)^{-1} & \text { if } p \equiv 1(\bmod 4), \\
1 & \text { if } p \equiv 3(\bmod 4) .\end{cases}
\end{aligned}
$$

Noting (3.1) and Theorem 1.3(i) we get

$$
\begin{gathered}
\prod_{1 \leqslant j<k \leqslant(p-1) / 2}\left(\zeta^{a j^{2}}-\zeta^{a k^{2}}\right)^{2} \\
=(-1)^{(p-1)(p-3) / 8} p^{(p-3) / 4} \times \begin{cases}\varepsilon_{p}^{\left(\frac{a}{p}\right) h(p)} & \text { if } p \equiv 1(\bmod 4), \\
1 & \text { if } p \equiv 3(\bmod 4) .\end{cases}
\end{gathered}
$$


QUADRATIC RESIDUES AND RELATED PERMUTATIONS AND IDENTITIES 25

Thus $(1.14)$ holds when $p \equiv 1(\bmod 4)$.

Below we suppose $p \equiv 3(\bmod 4)$ and want to show (1.15). By (3.11) with $a=1$, for some $\varepsilon \in\{ \pm 1\}$, we have

$$
\prod_{1 \leqslant j<k \leqslant(p-1) / 2}\left(\zeta^{j^{2}}-\zeta^{k^{2}}\right)=\varepsilon(\sqrt{p} i)^{(p-3) / 4} .
$$

In view of (3.7), this yields that

$$
\prod_{1 \leqslant j<k \leqslant(p-1) / 2}\left(\zeta^{a j^{2}}-\zeta^{a k^{2}}\right)=\varepsilon \varphi_{a}(\sqrt{p} i)^{(p-3) / 4}=\varepsilon\left(\left(\frac{a}{p}\right) \sqrt{p} i\right)^{(p-3) / 4} .
$$

As $i^{(p-3) / 4}=(-1)^{(p-7) / 8} i$ if $p \equiv 7(\bmod 8)$, we obtain $(1.15)$ from $(3.12)$ provided that

$$
\varepsilon= \begin{cases}1 & \text { if } p \equiv 3(\bmod 8) \\ (-1)^{(h(-p)+1) / 2} & \text { if } p \equiv 7(\bmod 8)\end{cases}
$$

Now it remains to show (3.13). By (3.12), for any $r=1, \ldots,(p-1) / 2$ we have

$$
\prod_{1 \leqslant j<k \leqslant(p-1) / 2}\left(\zeta^{r^{2} j^{2}}-\zeta^{r^{2} k^{2}}\right)=\varepsilon(\sqrt{p} i)^{(p-3) / 4}
$$

on the other hand,

$$
\prod_{1 \leqslant j<k \leqslant(p-1) / 2}\left(\zeta^{r^{2} j^{2}}-\zeta^{r^{2} k^{2}}\right)=\zeta^{r^{2} \sum_{1 \leqslant j<k \leqslant(p-1) / 2} j^{2}} \prod_{1 \leqslant j<k \leqslant(p-1) / 2}\left(1-\zeta^{r^{2}\left(k^{2}-j^{2}\right)}\right) .
$$

Combining these and noting (3.10) and (1.13), we find that

$$
\begin{aligned}
\left(\varepsilon(\sqrt{p} i)^{(p-3) / 4}\right)^{(p-1) / 2} & =\prod_{1 \leqslant j<k \leqslant(p-1) / 2} \prod_{r=1}^{(p-1) / 2}\left(1-\zeta^{\left(k^{2}-j^{2}\right) r^{2}}\right) \\
& =\prod_{1 \leqslant j<k \leqslant(p-1) / 2}\left((-1)^{(h(-p)+1) / 2}\left(\frac{k^{2}-j^{2}}{p}\right) \sqrt{p} i\right) .
\end{aligned}
$$

Therefore

$$
\varepsilon^{(p-1) / 2}=(-1)^{\frac{h(-p)+1}{2} \cdot \frac{(p-1)(p-3)}{8}} \prod_{1 \leqslant j<k \leqslant(p-1) / 2}\left(\frac{k^{2}-j^{2}}{p}\right)=(-1)^{\frac{h(-p)+1}{2} \cdot \frac{p-3}{4}}
$$

with the help of (1.5). This proves the desired (3.13) since $\varepsilon=\varepsilon^{(p-1) / 2}$.

The proof of Theorem 1.3 is now complete. 


\section{Proofs of Theorems 1.4 And 1.5}

Lemma 4.1. Let $p$ be any odd prime. Then

$$
\sum_{1 \leqslant j<k \leqslant(p-1) / 2}\left(j^{2}+k^{2}\right) \equiv \begin{cases}p(\bmod 2 p) & \text { if } p \equiv 5(\bmod 8), \\ 0(\bmod 2 p) & \text { otherwise. }\end{cases}
$$

Proof. Since

$$
\begin{aligned}
& 2 \sum_{1 \leqslant j<k \leqslant(p-1) / 2}\left(j^{2}+k^{2}\right)+\sum_{k=1}^{(p-1) / 2}\left(k^{2}+k^{2}\right) \\
& =\sum_{j=1}^{(p-1) / 2} \sum_{k=1}^{(p-1) / 2}\left(j^{2}+k^{2}\right)=(p-1) \sum_{k=1}^{(p-1) / 2} k^{2} \text {, }
\end{aligned}
$$

we have

$$
\sum_{1 \leqslant j<k \leqslant(p-1) / 2}\left(j^{2}+k^{2}\right)=\frac{p-3}{2} \sum_{k=1}^{(p-1) / 2} k^{2}=\frac{p-3}{2} \cdot \frac{p^{2}-1}{24} p \equiv 0 \quad(\bmod p) .
$$

Note that

$$
\frac{p-3}{2} \cdot \frac{p^{2}-1}{24} \equiv \begin{cases}(p-1) / 4(\bmod 2) & \text { if } p \equiv 1(\bmod 4) \\ 0(\bmod 2) & \text { if } p \equiv 3(\bmod 4)\end{cases}
$$

Therefore (4.1) holds.

Proof of Theorem 1.4. For $1 \leqslant j<k \leqslant(p-1) / 2$, clearly

$$
\left\{j^{2}\right\}_{p}>\left\{k^{2}\right\}_{p} \Longleftrightarrow \cot \pi \frac{j^{2}}{p}-\cot \pi \frac{k^{2}}{p}<0
$$

and

$$
\left\{k^{2}-j^{2}\right\}_{p}>\frac{p}{2} \Longleftrightarrow \sin 2 \pi \frac{k^{2}-j^{2}}{p}<0 \Longleftrightarrow \csc 2 \pi \frac{k^{2}-j^{2}}{p}<0 .
$$

So (1.20) follows from (1.21) and we only need to show part (ii) of Theorem 1.4 .

As (1.21) holds trivially for $p=3$, below we assume $p>3$.

For $1 \leqslant j<k \leqslant(p-1) / 2$, clearly

$$
\begin{aligned}
\sin \pi \frac{a\left(k^{2}-j^{2}\right)}{p} & =\frac{e^{i \pi a\left(k^{2}-j^{2}\right) / p}-e^{-i \pi a\left(k^{2}-j^{2}\right) / p}}{2 i} \\
& =\frac{i}{2} e^{-i \pi a\left(k^{2}+j^{2}\right) / p}\left(e^{2 \pi i a j^{2} / p}-e^{2 \pi i a k^{2} / p}\right) .
\end{aligned}
$$


Combining this with Lemma 4.1, we see that

$$
\begin{aligned}
& \prod_{1 \leqslant j<k \leqslant(p-1) / 2} \sin \pi \frac{a\left(k^{2}-j^{2}\right)}{p} \\
= & (-1)^{a \frac{p+1}{2}\left\lfloor\frac{p-1}{4}\right\rfloor}\left(\frac{i}{2}\right)^{(p-1)(p-3) / 8} \prod_{1 \leqslant j<k \leqslant(p-1) / 2}\left(e^{2 \pi i a j^{2} / p}-e^{2 \pi i a k^{2} / p}\right) .
\end{aligned}
$$

For any real numbers $\theta_{1}, \theta_{2} \notin \mathbb{Z}$, clearly

$$
\cot \pi \theta_{1}-\cot \pi \theta_{2}=\frac{\cos \pi \theta_{1}}{\sin \pi \theta_{1}}-\frac{\cos \pi \theta_{2}}{\sin \pi \theta_{2}}=\frac{\sin \pi\left(\theta_{2}-\theta_{1}\right)}{\sin \pi \theta_{1} \sin \pi \theta_{2}}
$$

Thus

$$
\begin{aligned}
& \prod_{1 \leqslant j<k \leqslant(p-1) / 2} \frac{\sin \pi a\left(k^{2}-j^{2}\right) / p}{\cot \pi a j^{2} / p-\cot \pi a k^{2} / p} \\
= & \prod_{1 \leqslant j<k \leqslant(p-1) / 2} \sin \pi \frac{a j^{2}}{p} \sin \pi \frac{a k^{2}}{p}=\prod_{k=1}^{(p-1) / 2}\left(\sin \pi \frac{a k^{2}}{p}\right)^{|\{1 \leqslant j \leqslant(p-1) / 2: j \neq k\}|}
\end{aligned}
$$

and hence by (1.16) we have

$$
\begin{aligned}
& \prod_{1 \leqslant j<k \leqslant(p-1) / 2} \sin \pi \frac{a\left(k^{2}-j^{2}\right)}{p} / \prod_{1 \leqslant j<k \leqslant(p-1) / 2}\left(\cot \pi \frac{a j^{2}}{p}-\cot \pi \frac{a k^{2}}{p}\right) \\
= & \left(\frac{p}{2^{p-1}}\right)^{(p-3) / 4} \times \begin{cases}(-1)^{(a-1)(p-1) / 4} \varepsilon_{p}^{-\left(\frac{a}{p}\right) \frac{p-3}{2} h(p)} & \text { if } p \equiv 1(\bmod 4), \\
1 & \text { if } p \equiv 3(\bmod 4) .\end{cases}
\end{aligned}
$$

So it suffices to determine $\prod_{1 \leqslant j<k \leqslant(p-1) / 2} \sin \pi a\left(k^{2}-j^{2}\right) / p$.

Case 1. $p \equiv 3(\bmod 4)$.

In this case, by combining (4.2) and (1.15) we get

$$
\begin{aligned}
& \prod_{1 \leqslant j<k \leqslant(p-1) / 2} \sin \pi \frac{a\left(k^{2}-j^{2}\right)}{p} \\
= & \left(\frac{p}{2^{p-1}}\right)^{(p-3) / 8} \times \begin{cases}1 & \text { if } p \equiv 3(\bmod 8), \\
(-1)^{(h(-p)+1) / 2}\left(\frac{a}{p}\right) & \text { if } p \equiv 7(\bmod 8) .\end{cases}
\end{aligned}
$$

Thus (1.21) is valid with the help of (4.3).

Case $2 . p \equiv 1(\bmod 4)$.

In this case, combining (4.2) with (1.14) we obtain

$$
\prod_{1 \leqslant j<k \leqslant(p-1) / 2} \sin ^{2} \pi \frac{a\left(k^{2}-j^{2}\right)}{p}=\left(\frac{p}{2^{p-1}}\right)^{(p-3) / 4} \varepsilon_{p}^{\left(\frac{a}{p}\right) h(p)}
$$


and hence

$$
\prod_{1 \leqslant j<k \leqslant(p-1) / 2} \csc \pi \frac{a\left(k^{2}-j^{2}\right)}{p}= \pm\left(2^{p-1} p^{-1}\right)^{(p-3) / 8} \varepsilon_{p}^{-\left(\frac{a}{p}\right) h(p) / 2}
$$

In view of $(4.3)$, we have

$$
\begin{aligned}
& \prod_{1 \leqslant j<k \leqslant(p-1) / 2}\left(\sin \pi \frac{a\left(k^{2}-j^{2}\right)}{p}\right)\left(\cot \pi \frac{a j^{2}}{p}-\cot \frac{a k^{2}}{p}\right) \\
= & (-1)^{(a-1)(p-1) / 4}\left(2^{p-1} p^{-1}\right)^{(p-3) / 4} \varepsilon_{p}^{\left(\frac{a}{p}\right) \frac{p-3}{2} h(p)} \prod_{1 \leqslant j<k \leqslant(p-1) / 2} \sin ^{2} \pi \frac{a\left(k^{2}-j^{2}\right)}{p} .
\end{aligned}
$$

Combining this with (4.4) we immediately get the first equality in (1.22).

The proof of Theorem 1.4 is now complete.

Proof of Theorem 1.5. (1.23) is trivial for $p=3$. Below we assume $p>3$. In view of (4.2),

$$
\begin{aligned}
\prod_{1 \leqslant j<k \leqslant(p-1) / 2}\left(2 \cos \pi \frac{a\left(k^{2}-j^{2}\right)}{p}\right) & =\prod_{1 \leqslant j<k \leqslant(p-1) / 2} \frac{\sin \pi(2 a)\left(k^{2}-j^{2}\right) / p}{\sin \pi a\left(k^{2}-j^{2}\right) / p} \\
& =(-1)^{a \frac{p+1}{2}\left\lfloor\frac{p-1}{4}\right\rfloor} \prod_{1 \leqslant j<k \leqslant(p-1) / 2}\left(\zeta^{a j^{2}}+\zeta^{a k^{2}}\right) .
\end{aligned}
$$

So we have the first equality in (1.23). On the other hand, by Theorem 1.4(ii) we have

$$
\prod_{1 \leqslant j<k \leqslant(p-1) / 2} \frac{\csc \pi a\left(k^{2}-j^{2}\right) / p}{\csc \pi(2 a)\left(k^{2}-j^{2}\right) / p}= \begin{cases}1 & \text { if } p \equiv 3(\bmod 4) \\ \pm \varepsilon_{p}^{\left(\frac{a}{p}\right) h(p)\left(\left(\frac{2}{p}\right)-1\right) / 2} & \text { if } p \equiv 1(\bmod 4)\end{cases}
$$

Therefore (1.23) holds.

The proof of Theorem 1.5 is now complete.

\section{Proof of Theorem 1.6}

Lemma 5.1. Let $p$ be an odd prime. Then

$$
\frac{1}{p} \sum_{\substack{1 \leqslant j<k \leqslant(p-1) / 2 \\ p \mid j^{2}+k^{2}}}\left(j^{2}+k^{2}\right) \equiv \begin{cases}1(\bmod 2) & \text { if } p \equiv 5(\bmod 8) \\ 0(\bmod 2) & \text { otherwise }\end{cases}
$$

Proof. If $p \equiv 3(\bmod 4)$, then $\left(\frac{-1}{p}\right)=-1$ and $j^{2}+k^{2} \not \equiv 0(\bmod p)$ for any $j, k=1, \ldots,(p-1) / 2$. So $(5.1)$ is trivial in the case $p \equiv 3(\bmod 4)$. 
Now assume that $p \equiv 1(\bmod 4)$. Then $q^{2} \equiv-1(\bmod p)$ for some $q \in \mathbb{Z}$. For each $j=1, \ldots,(p-1) / 2$ let $j_{*}$ be the unique integer $r \in\{1, \ldots,(p-1) / 2\}$ with $q j$ congruent to $r$ or $-r$ modulo $p$. Clearly, $\left\{j_{*}: 1 \leqslant j \leqslant(p-1) / 2\right\}=$ $\{1, \ldots,(p-1) / 2\}$. Thus

$$
\begin{aligned}
\sum_{\substack{1 \leqslant j<k \leqslant(p-1) / 2 \\
p \mid j^{2}+k^{2}}}\left(j^{2}+k^{2}\right) & =\frac{1}{2} \sum_{\substack{j, k=1 \\
p \mid j^{2}+k^{2}}}^{(p-1) / 2}\left(j^{2}+k^{2}\right) \\
& =\frac{1}{2} \sum_{j=1}^{(p-1) / 2}\left(j^{2}+j_{*}^{2}\right)=\sum_{k=1}^{(p-1) / 2} k^{2}=\frac{p^{2}-1}{24} p
\end{aligned}
$$

and hence

$$
\frac{1}{p} \sum_{\substack{1 \leqslant j<k \leqslant(p-1) / 2 \\ p \mid j^{2}+k^{2}}}\left(j^{2}+k^{2}\right)=\frac{p^{2}-1}{24} \equiv \frac{p^{2}-1}{8} \equiv \frac{p-1}{4} \quad(\bmod 2) .
$$

Therefore (5.1) holds.

Proof of Theorem 1.6(i). Let $\zeta=e^{2 \pi i / p}$. As

$$
\sum_{\substack{1 \leqslant j<k \leqslant(p-1) / 2 \\ p \nmid j^{2}+k^{2}}}\left(j^{2}+k^{2}\right) \equiv 0 \quad(\bmod 2 p)
$$

by Lemmas 4.1 and 5.1 , we have

$$
\begin{aligned}
\prod_{\substack{1 \leqslant j<k \leqslant(p-1) / 2 \\
p \nmid j^{2}+k^{2}}} \sin \pi \frac{a\left(j^{2}+k^{2}\right)}{p} & =\prod_{\substack{1 \leqslant j<k \leqslant(p-1) / 2 \\
p \nmid j^{2}+k^{2}}} \frac{-e^{-i \pi a\left(j^{2}+k^{2}\right) / p}}{2 i}\left(1-\zeta^{a\left(j^{2}+k^{2}\right)}\right) \\
& =\left(\frac{i}{2}\right)^{\left|\left\{(j, k): 1 \leqslant j<k \leqslant(p-1) / 2 \& p \nmid j^{2}+k^{2}\right\}\right|} f(a),
\end{aligned}
$$

where

$$
f(a):=\prod_{n=1}^{p-1}\left(1-\zeta^{a n}\right)^{r(n)}
$$

with $r(n)$ defined as in Lemma 2.3. Note that

$$
\begin{aligned}
& \left|\left\{(j, k): 1 \leqslant j<k \leqslant \frac{p-1}{2} \& p \nmid j^{2}+k^{2}\right\}\right| \\
= & \left(\begin{array}{c}
(p-1) / 2 \\
2
\end{array}\right)-r(0)=\frac{p-1}{2}\left\lfloor\frac{p-3}{4}\right\rfloor
\end{aligned}
$$


with the help of (2.6). So

$$
\prod_{\substack{1 \leqslant j<k \leqslant(p-1) / 2 \\ p \nmid j^{2}+k^{2}}} \sin \pi \frac{a\left(j^{2}+k^{2}\right)}{p}=\left(\frac{i}{2}\right)^{\frac{p-1}{2}\left\lfloor\frac{p-3}{4}\right\rfloor} f(a) .
$$

By (2.7), (3.1) and Theorem 1.3(i), we have

$$
\begin{aligned}
f(a) & =p^{\lfloor(p+1) / 8\rfloor} \prod_{\substack{n=1 \\
\left(\frac{n}{p}\right)=1}}^{p-1}\left(1-\zeta^{a n}\right)^{-\left(1+\left(\frac{2}{p}\right)\right) / 2} \\
& = \begin{cases}p^{\lfloor(p+1) / 8\rfloor} & \text { if } p \equiv 3,5(\bmod 8), \\
p^{\lfloor(p+1) / 8\rfloor-1 / 2} \varepsilon_{p}^{\left(\frac{a}{p}\right) h(p)} & \text { if } p \equiv 1(\bmod 8), \\
(-1)^{(h(-p)-1) / 2}\left(\frac{a}{p}\right) p^{\lfloor(p+1) / 8\rfloor-1 / 2} i & \text { if } p \equiv 7(\bmod 8) .\end{cases}
\end{aligned}
$$

Combining this with (5.3) we immediately get (1.25).

In light of (1.25) and (5.2),

$$
\begin{aligned}
\prod_{\substack{1 \leqslant j<k \leqslant(p-1) / 2 \\
p \nmid j^{2}+k^{2}}} \cos \pi \frac{a\left(j^{2}+k^{2}\right)}{p} & =\prod_{\substack{1 \leqslant j<k \leqslant(p-1) / 2 \\
p \nmid j^{2}+k^{2}}} \frac{\sin \pi(2 a)\left(j^{2}+k^{2}\right) / p}{2 \sin \pi a\left(j^{2}+k^{2}\right) / p} \\
& =2^{-\left|\left\{(j, k): 1 \leqslant j<k \leqslant(p-1) / 2 \& p \nmid j^{2}+k^{2}\right\}\right|} \\
& =2^{-\frac{p-1}{2}\left\lfloor\frac{p-3}{4}\right\rfloor .}
\end{aligned}
$$

Note also that

$$
\begin{gathered}
\prod_{\substack{1 \leqslant j<k \leqslant(p-1) / 2 \\
p \mid j^{2}+k^{2}}} \cos \pi \frac{a\left(j^{2}+k^{2}\right)}{p} \\
=(-1)^{\sum_{1 \leqslant j<k \leqslant(p-1) / 2 \& p \mid j^{2}+k^{2}} a\left(j^{2}+k^{2}\right) / p}=(-1)^{a \frac{p+1}{2}\left\lfloor\frac{p-1}{4}\right\rfloor}
\end{gathered}
$$

by Lemma 5.1. Therefore (1.26) holds.

Observe that

$\prod_{\substack{1 \leqslant j<k \leqslant(p-1) / 2 \\ p \nmid j^{2}+k^{2}}}\left(\cot \pi \frac{a j^{2}}{p}+\cot \pi \frac{a k^{2}}{p}\right)=\prod_{\substack{1 \leqslant j<k \leqslant(p-1) / 2 \\ p \nmid j^{2}+k^{2}}} \frac{\sin \pi a\left(j^{2}+k^{2}\right) / p}{\left(\sin \pi a j^{2} / p\right)\left(\sin \pi a k^{2} / p\right)}$

and

$$
\prod_{\substack{1 \leqslant j<k \leqslant(p-1) / 2 \\ p \nmid j^{2}+k^{2}}}\left(\sin \pi \frac{a j^{2}}{p}\right)\left(\sin \pi \frac{a k^{2}}{p}\right)=\prod_{k=1}^{(p-1) / 2}\left(\sin \pi \frac{a k^{2}}{p}\right)^{\left(p-\left(\frac{-1}{p}\right)-4\right) / 2} .
$$

Combining these with (1.25) and (1.16), we obtain the desired (1.27). This concludes the proof of Theorem 1.6(i). 
QUADRATIC RESIDUES AND RELATED PERMUTATIONS AND IDENTITIES 31

Lemma 5.2. Let $p>3$ be a prime and let $a, b, c \in \mathbb{Z}$ with $p \nmid a$. Then

$$
\sum_{1 \leqslant j<k \leqslant p-1}\left(a j^{2}+b j k+c k^{2}\right) \equiv 0 \quad(\bmod p)
$$

and also

$$
\frac{1}{p} \sum_{1 \leqslant j<k \leqslant p-1}\left(a j^{2}+b j k+c k^{2}\right) \equiv a \frac{p-1}{2}+b \frac{(p-1)(p-3)}{8} \quad(\bmod 2) .
$$

Proof. Let $\Delta=b^{2}-4 a c$. In view of (3.10), we have

$$
\begin{aligned}
& \sum_{1 \leqslant j<k \leqslant p-1}\left(a j^{2}+b j k+c k^{2}\right) \\
= & \sum_{1 \leqslant j<k \leqslant(p-1) / 2}\left(a j^{2}+b j k+c k^{2}\right) \\
& +\sum_{1 \leqslant k \leqslant(p-1) / 2} \sum_{k<j \leqslant p-1}\left(a(p-j)^{2}+b(p-j)(p-k)+c(p-k)^{2}\right) \\
\equiv & \sum_{k=1}^{(p-1) / 2}\left(\sum_{j=0}^{p-1}\left(a j^{2}+b j k+c k^{2}\right)-c k^{2}-(a+b+c) k^{2}\right) \\
\equiv & \sum_{k=1}^{(p-1) / 2} \sum_{j=0} \frac{1}{4 a}\left((2 a j+b k)^{2}-\Delta k^{2}\right) \equiv \sum_{k=1}^{(p-1) / 2} \frac{1}{4 a} \sum_{r=0}^{p-1} r^{2} \equiv 0(\bmod p) .
\end{aligned}
$$

This proves (5.4).

Observe that

$$
\begin{aligned}
& \sum_{1 \leqslant j<k \leqslant p-1}\left(a j^{2}+b j k+c k^{2}\right) \\
\equiv & \sum_{1 \leqslant j<k \leqslant p-1}(a j+c k)+b \sum_{1 \leqslant j<k \leqslant(p-1) / 2}(2 j-1)(2 k-1) \\
\equiv & \sum_{k=1}^{p-1}\left(\sum_{0<j<k} a j+c k(k-1)\right)+b\left(\begin{array}{c}
(p-1) / 2 \\
2
\end{array}\right) \\
\equiv & \sum_{k=1}^{p-1} \frac{a}{2}\left(k^{2}-k\right)+b \frac{(p-1)(p-3)}{8}(\bmod 2)
\end{aligned}
$$

and hence

$$
\begin{aligned}
& \sum_{1 \leqslant j<k \leqslant p-1}\left(a j^{2}+b j k+c k^{2}\right)-b \frac{(p-1)(p-3)}{8} \\
\equiv & \frac{a}{2}\left(\frac{(p-1) p(2 p-1)}{6}-\frac{(p-1) p}{2}\right)=a \frac{p(p-1)(p-2)}{6} \equiv a \frac{p-1}{2}(\bmod 2) .
\end{aligned}
$$


Therefore (5.5) also holds.

Proof of Theorem 1.6(ii). By Lemma 2.4,

$$
\begin{aligned}
& \mid\left\{(j, k): 1 \leqslant j<k \leqslant p-1 \text { and } p \nmid a j^{2}+b j k+c k^{2}\right\} \mid \\
= & \left(\begin{array}{c}
p-1 \\
2
\end{array}\right)-\frac{p-1}{2}\left(1+\left(\frac{\Delta}{p}\right)\right)=\frac{p-1}{2}\left(p-3-\left(\frac{\Delta}{p}\right)\right) .
\end{aligned}
$$

Let $\zeta=e^{2 \pi i / p}$. Then

$$
\begin{aligned}
& \prod_{\substack{1 \leqslant j<k \leqslant p-1 \\
p \nmid a j^{2}+b j k+c k^{2}}} \sin \pi \frac{a j^{2}+b j k+c k^{2}}{p} \\
= & \prod_{\substack{1 \leqslant j<k \leqslant p-1 \\
p \nmid a j^{2}+b j k+c k^{2}}} \frac{-e^{-i \pi\left(a j^{2}+b j k+c k^{2}\right) / p}}{2 i}\left(1-\zeta^{\left.a j^{2}+b j k+c k^{2}\right)}\right. \\
= & \left(\frac{i}{2}\right)^{\frac{p-1}{2}\left(p-3-\left(\frac{\Delta}{p}\right)\right)}(-1)^{a(p-1) / 2+b(p-1)(p-3) / 8-m} \\
& \prod_{\substack{1 \leqslant j<k \leqslant p-1 \\
p \nmid a j^{2}+b j k+c k^{2}}}\left(1-\zeta^{a j^{2}+b j k+c k^{2}}\right)
\end{aligned}
$$

with the help of Lemma 5.2. In view of Lemma 2.4, (3.1) and Theorem 1.3(i), we have

$$
\begin{aligned}
& \prod_{\substack{1 \leqslant j<k \leqslant p-1 \\
p \nmid a j^{2}+b j k+c k^{2}}}\left(1-\zeta^{a j^{2}+b j k+c k^{2}}\right) \\
= & \frac{\prod_{n=1}^{p-1}\left(1-\zeta^{n}\right)^{\left(p-3-\left(\frac{\Delta}{p}\right)+\left(1-p+p\left(\frac{\Delta}{p}\right)^{2}\right)\left(\frac{a}{p}\right)+\left(\frac{c}{p}\right)+\left(\frac{a+b+c}{p}\right)\right) / 2}}{\prod_{n=1}^{p-1}\left(1-\zeta^{n}\right)^{\left(\left(1-p+p\left(\frac{\Delta}{p}\right)^{2}\right)\left(\frac{a}{p}\right)+\left(\frac{c}{p}\right)+\left(\frac{a+b+c}{p}\right)\right)\left(1+\left(\frac{n}{p}\right)\right) / 2}} \\
= & \frac{p^{\left(p-3-\left(\frac{\Delta}{p}\right)+\left(1-p+p\left(\frac{\Delta}{p}\right)^{2}\right)\left(\frac{a}{p}\right)+\left(\frac{c}{p}\right)+\left(\frac{a+b+c}{p}\right)\right) / 2}}{\prod_{k=1}^{(p-1) / 2}\left(1-\zeta^{2}\right)^{\left(1-p+p\left(\frac{\Delta}{p}\right)^{2}\right)\left(\frac{a}{p}\right)+\left(\frac{c}{p}\right)+\left(\frac{a+b+c}{p}\right)}} \\
= & p^{\left(p-3-\left(\frac{\Delta}{p}\right) / 2\right.} \\
& \times \begin{cases}\varepsilon_{p}^{h(p)\left(\left(1-p+p\left(\frac{\Delta}{p}\right)^{2}\right)\left(\frac{a}{p}\right)+\left(\frac{c}{p}\right)+\left(\frac{a+b+c}{p}\right)\right)} & \text { if } p \equiv 1(\bmod 4), \\
\left((-1)^{(h(-p)-1) / 2} i\right)^{\left(1-p+p\left(\frac{\Delta}{p}\right)^{2}\right)\left(\frac{a}{p}\right)+\left(\frac{c}{p}\right)+\left(\frac{a+b+c}{p}\right)} & \text { if } p \equiv 3(\bmod 4) .\end{cases}
\end{aligned}
$$

Therefore

$$
\begin{aligned}
&(-1)^{m} \prod_{\substack{1 \leqslant j<k \leqslant p-1 \\
p \nmid a j^{2}+b j k+c k^{2}}} \sin \pi \frac{a j^{2}+b j k+c k^{2}}{p} \\
&=(-1)^{a(p-1) / 2+b(p-1)(p-3) / 8} i^{\frac{p-1}{2}\left(p-3-\left(\frac{\Delta}{p}\right)\right)}\left(\frac{p}{2^{p-1}}\right)^{\left(p-3-\left(\frac{\Delta}{p}\right)\right) / 2} \\
& \times \begin{cases}\varepsilon_{p}^{h(p)\left(\left(1-p+p\left(\frac{\Delta}{p}\right)^{2}\right)\left(\frac{a}{p}\right)+\left(\frac{c}{p}\right)+\left(\frac{a+b+c}{p}\right)\right)} & \text { if } p \equiv 1(\bmod 4), \\
(-1)^{\left(\frac{\Delta}{p}\right) \frac{h(-p)-1}{2}+\frac{1-p}{2}\left(\frac{a}{p}\right)+\frac{1}{2}\left(\left(\frac{c}{p}\right)+\left(\frac{a+b+c}{p}\right)\right)} i^{p\left(\frac{\Delta}{p}\right)^{2}\left(\frac{a}{p}\right)} & \text { if } p \equiv 3(\bmod 4) .\end{cases}
\end{aligned}
$$


QUADRATIC RESIDUES AND RELATED PERMUTATIONS AND IDENTITIES 33

It is easy to see that this implies (1.29).

Clearly,

$$
\prod_{\substack{1 \leqslant j<k \leqslant p-1 \\ p \mid a j^{2}+b j k+c k^{2}}} \cos \pi \frac{a j^{2}+b j k+c k^{2}}{p}=\prod_{\substack{1 \leqslant j<k \leqslant p-1 \\ p \mid a j^{2}+b j k+c k^{2}}}(-1)^{\left(a j^{2}+b j k+c k^{2}\right) / p}=(-1)^{m} .
$$

On the other hand, by (5.6) we have

$$
\begin{aligned}
& 2^{\frac{p-1}{2}\left(p-3-\left(\frac{\Delta}{p}\right)\right)} \prod_{\substack{1 \leqslant j<k \leqslant p-1 \\
p \nmid a j^{2}+b j k+c k^{2}}} \cos \pi \frac{a j^{2}+b j k+c k^{2}}{p} \\
= & \prod_{\substack{1 \leqslant j<k \leqslant p-1 \\
p \nmid a j^{2}+b j k+c k^{2}}} \frac{\sin \pi\left(2 a j^{2}+2 b j k+2 c k^{2}\right) / p}{\sin \pi\left(a j^{2}+b j k+c k^{2}\right) / p} .
\end{aligned}
$$

Combining these with (1.29) we immediately obtain the desired (1.30).

In view of the above, we have completed the proof of Theorem 1.6(ii).

\section{Some COnjectures}

We are unable to determine the parities of $s(p)$ and $t(p)$ (defined by (1.18) and $(1.19))$ for a general prime $p \equiv 1(\bmod 4)$. However, in contrast with (1.20), we formulate the following conjecture.

Conjecture 6.1. For any prime $p \equiv 1(\bmod 4)$, we have

$$
s(p)+t(p) \equiv\left|\left\{1 \leqslant k<\frac{p}{4}:\left(\frac{k}{p}\right)=1\right\}\right| \quad(\bmod 2) .
$$

For any positive odd number $n$ and integer $k$, we let $R(k, n)$ denote the unique $r \in\{0, \ldots,(n-1) / 2\}$ with $k$ congruent to $r$ or $-r$ modulo $n$. For example,

$$
R\left(1^{2}, 11\right)=1, R\left(2^{2}, 11\right)=4, R\left(3^{2}, 11\right)=2, R\left(4^{2}, 11\right)=5, R\left(5^{2}, 11\right)=3 .
$$

Motivated by Theorem 1.4 and Corollary 1.3, we pose the following conjecture.

Conjecture 6.2. Let $p$ be an odd prime, and let $a \in \mathbb{Z}$ with $p \nmid a$. Then

$$
\mid\left\{(i, j): 1 \leqslant i<j \leqslant \frac{p-1}{2} \text { and } R\left(a i^{2}, p\right)>R\left(a j^{2}, p\right)\right\} \mid \equiv\left\lfloor\frac{p+1}{8}\right\rfloor \quad(\bmod 2) \text {, }
$$

and

$$
\begin{aligned}
& (-1)^{\left|\left\{(i, j): 1 \leqslant i<j \leqslant(p-1) / 2 \& R\left(a i^{2}, p\right)+R\left(a j^{2}, p\right)>p / 2\right\}\right|} \\
= & \begin{cases}(-1)^{\left|\left\{1 \leqslant k<\frac{p}{4}:\left(\frac{k}{p}\right)=-1\right\}\right|}\left(\frac{a}{p}\right)^{\left(1-\left(\frac{2}{p}\right)\right) / 2} & \text { if } p \equiv 1(\bmod 4), \\
1 & \text { if } p \equiv 3(\bmod 4) .\end{cases}
\end{aligned}
$$

Remark 6.1. We have verified (6.2) and (6.3) with $a=1$ for all odd primes $p<20000$. 
Conjecture 6.3. Let $p>3$ be a prime. If $p \equiv 3(\bmod 4)$, then

$$
\begin{aligned}
& (-1)^{\left|\left\{(j, k): 1 \leqslant j<k \leqslant(p-1) / 2 \&\{j(j+1) / 2\}_{p}>\{k(k+1) / 2\}_{p}\right\}\right|} \\
& =(-1)^{\frac{h(-p)+1}{2}+\left|\left\{1 \leqslant k \leqslant\left\lfloor\frac{p+1}{8}\right\rfloor:\left(\frac{k}{p}\right)=1\right\}\right|} .
\end{aligned}
$$

Also,

$$
\begin{aligned}
& (-1)^{\left|\left\{(j, k): 1 \leqslant j<k \leqslant(p-1) / 2 \&\{j(j+1) / 2\}_{p}+\{k(k+1) / 2\}_{p}>p\right\}\right|} \\
& = \begin{cases}(-1)^{(p-1) / 8} & \text { if } p \equiv 1(\bmod 8), \\
(-1)^{\left|\left\{1 \leqslant k<\frac{p}{4}:\left(\frac{k}{p}\right)=-1\right\}\right|} & \text { if } p \equiv 5(\bmod 8), \\
(-1)^{\frac{h(-p)+1}{2}+\left|\left\{1 \leqslant k \leqslant\left\lfloor\frac{p+1}{8}\right\rfloor:\left(\frac{k}{p}\right)=-1\right\}\right|} & \text { if } p \equiv 3(\bmod 4) .\end{cases}
\end{aligned}
$$

Conjecture 6.4. Let $p$ be an odd prime. If $p \equiv 3(\bmod 4)$, then

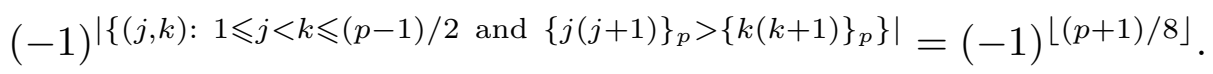

Also,

$$
\begin{aligned}
& (-1)^{\left|\left\{(j, k): 1 \leqslant j<k \leqslant(p-1) / 2 \&\{j(j+1)\}_{p}+\{k(k+1)\}_{p}>p\right\}\right|} \\
& = \begin{cases}(-1)^{\lfloor(p-1) / 8\rfloor} & \text { if } p \equiv 1(\bmod 4), \\
(-1)^{(h(-p)+1) / 2} & \text { if } p>3 \& p \equiv 3(\bmod 8), \\
1 & \text { if } p \equiv 7(\bmod 8) .\end{cases}
\end{aligned}
$$

Conjecture 6.5. (i) For any prime $p \equiv 5(\bmod 6)$, we have

$$
\left|\left\{1 \leqslant k \leqslant \frac{p-1}{2}:\left\{k^{3}\right\}_{p}>\frac{p}{2}\right\}\right|-\frac{p+1}{6} \in\{2 n: n=0,1,2, \ldots\}
$$

and

$$
\mid\left\{(j, k): 1 \leqslant j<k \leqslant p-1 \text { and }\left\{j^{3}\right\}_{p}>\left\{k^{3}\right\}_{p}\right\} \mid \equiv \frac{p+1}{6} \quad(\bmod 2) .
$$

(ii) For any integer $m>1$, we have

$$
\left|\left\{1 \leqslant k \leqslant \frac{p-1}{2}:\left\{k^{m}\right\}_{p}>\frac{p}{2}\right\}\right| \sim \frac{p}{4}
$$

as $p \rightarrow \infty$, where $p$ is an odd prime.

Remark 6.2. Let $p$ be a prime with $p \equiv 5(\bmod 6)$. The list $\left\{1^{3}\right\}_{p}, \ldots,\{(p-$ $\left.1)^{3}\right\}_{p}$ is a permutation of $1, \ldots, p-1$, for, if $1 \leqslant j<k \leqslant p-1$ then

$$
j^{3}-k^{3}=(j-k)\left(j^{2}+j k+k^{2}\right)=\frac{j-k}{4}\left((2 j+k)^{2}+3 k^{2}\right) \not \equiv 0 \quad(\bmod p) .
$$

See [S18, A320044] for some data related to (6.8). Note that (6.8) implies (6.9) since for any $1 \leqslant j<k \leqslant p-1$ we have $1 \leqslant p-k<p-j \leqslant p-1$ and

$$
\left(\left\{j^{3}\right\}_{p}-\left\{k^{3}\right\}_{p}\right)\left(\left\{(p-k)^{3}\right\}_{p}-\left\{(p-j)^{3}\right\}_{p}\right)>0 .
$$


QUADRATIC RESIDUES AND RELATED PERMUTATIONS AND IDENTITIES 35

Conjecture 6.6. Let $p$ be an odd prime. Then

$$
\begin{aligned}
& \mid\left\{(j, k): 1 \leqslant j<k \leqslant \frac{p-1}{2} \text { and }\left\{j^{4}\right\}_{p}>\left\{k^{4}\right\}_{p}\right\} \mid \\
\equiv & \left\lfloor\frac{p+1}{8}\right\rfloor+ \begin{cases}(h(-p)+1) / 2(\bmod 2) & \text { if } p \equiv 7(\bmod 8), \\
0(\bmod 2) & \text { otherwise. }\end{cases}
\end{aligned}
$$

Also,

$$
\begin{aligned}
& \mid\left\{(j, k): 1 \leqslant j<k \leqslant \frac{p-1}{2} \text { and }\left\{j^{8}\right\}_{p}>\left\{k^{8}\right\}_{p}\right\} \mid \\
\equiv & \begin{cases}\left|\left\{1 \leqslant k<\frac{p}{4}:\left(\frac{k}{p}\right)=1\right\}\right|(\bmod 2) & \text { if } p \equiv 1(\bmod 8), \\
0(\bmod 2) & \text { if } p \equiv 3(\bmod 8), \\
(p-5) / 8(\bmod 2) & \text { if } p \equiv 5(\bmod 8), \\
(h(-p)+1) / 2(\bmod 2) & \text { if } p \equiv 7(\bmod 8) .\end{cases}
\end{aligned}
$$

Remark 6.3. See [S18, A309012, A319882, A319894 and A319903] for related data or similar conjectures.

The following conjecture is motivated by Theorem 1.5.

Conjecture 6.7. Let $p$ be a prime with $p \equiv 1(\bmod 4)$, and let $\zeta=e^{2 \pi i / p}$. Let $a$ be an integer not divisible by $p$. Then

$$
\begin{aligned}
& (-1)^{\left|\left\{1 \leqslant k<p / 4:\left(\frac{k}{p}\right)=-1\right\}\right|} \prod_{1 \leqslant j<k \leqslant(p-1) / 2}\left(\zeta^{a j^{2}}+\zeta^{a k^{2}}\right) \\
= & \begin{cases}1 & \text { if } p \equiv 1(\bmod 8), \\
\left(\frac{a}{p}\right) \varepsilon_{p}^{-\left(\frac{a}{p}\right) h(p)} & \text { if } p \equiv 5(\bmod 8) .\end{cases}
\end{aligned}
$$

Remark 6.4. By K. S. Williams and J. D. Currie [WC], for any prime $p \equiv 1$ $(\bmod 8)$ we have

$$
2^{(p-1) / 4} \equiv(-1)^{\left|\left\{1 \leqslant k<p / 4:\left(\frac{k}{p}\right)=-1\right\}\right|} \quad(\bmod p) .
$$

The author [S19] studied the determinants of the matrices

$$
\left[\left(\frac{i^{2}+j^{2}}{p}\right)\right]_{1 \leqslant i, j \leqslant(p-1) / 2} \text { and }\left[\left(\frac{i^{2}+j^{2}}{p}\right)\right]_{0 \leqslant i, j \leqslant(p-1) / 2}
$$

where $p$ is an odd prime. Now we conclude this section with a conjecture involving determinants. 
Conjecture 6.8. Let $n>1$ be an odd integer. Then

$$
\operatorname{det}\left[R\left(i^{2} j^{2}, n\right)\right]_{1 \leqslant i, j \leqslant(n-1) / 2} \neq 0
$$

if and only if $n$ is a prime congruent to 3 modulo 4. Also,

$$
\operatorname{det}\left[\left\lfloor\frac{i^{2} j^{2}}{n}\right\rfloor\right]_{1 \leqslant i, j \leqslant(n-1) / 2} \neq 0
$$

if and only if $n$ is either 9 or a prime greater than 7 and congruent to 3 modulo 4 .

Acknowledgments. The author would like to thank the two referees for their helpful comments.

\section{REFERENCES}

[BEW] B. C. Berndt, R. J. Evans and K. S. Williams, Gauss and Jacobi Sums, John Wiley \& Sons, 1998.

[BC] A. Brunyate and P. L. Clark, Extending the Zolotarev-Frobenius approach to quadratic reciprocity, Ramanujan J. 37 (2015), 25-50.

[Ch] R. Chapman, Determinants of Legendre symbol matrices, Acta Arith. 115 (2004), 231-244.

[Co] H. Cohn, Advanced Number Theory, Dover Publ., New York, 1962.

$[\mathrm{DH}]$ W. Duke and K. Hopkins, Quadratic reciprocity in a finite group, Amer. Math. Monthly 112 (2005), 251-256.

[IR] K. Ireland and M. Rosen, A Classical Introduction to Modern Number Theory, 2nd Edition, Graduate Texts in Math., Vol. 84, Springer, New York, 1990.

[M61] L. J. Mordell, The congruence $((p-1) / 2) ! \equiv \pm 1(\bmod p)$, Amer. Math. Monthly 68 (1961), 145-146.

[P06] H. Pan, A remark on Zolotarev's theorem, preprint, arXiv:0601026, 2006.

[S18] Z.-W. Sun, Sequences A309012, A319311, A319882, A319894, A319903, A320044 in OEIS, http://oeis.org.

[S19] Z.-W. Sun, On some determinants with Legendre symbol entries, Finite Fields Appl. 56 (2019), 285-307.

[Sz] G. J. Szekely (ed.), Contests in Higher Mathematics, Springer, New York, 1996.

[WC] K. S. Williams and J. D. Currie, Class numbers and biquadratic reciprocity, Canad. J. Math. 34 (1982), 969-988.

[Z] G. Zolotarev, Nouvelle démonstration de la loi de réciprocité de Legendre, Nouv. Ann. Math. 11 (1872), 354-362. 